\title{
GJB2 (connexin 26) variants and nonsyndromic sensorineural hearing loss: A HuGE review
}

Aileen Kenneson, PhD, Kim Van Naarden Braun, MPH, and Coleen Boyle, PhD

\begin{abstract}
Despite the enormous heterogeneity of genetic hearing loss, variants in one locus, Gap Junction Beta 2 or GJB2 (connexin 26), account for up to $50 \%$ of cases of nonsyndromic sensorineural hearing loss in some populations. This article reviews genetic epidemiology studies of the alleles of GJB2, prevalence rates, genotype-phenotype relations, contribution to the incidence of hearing loss, and other issues related to the clinical validity of genetic testing for GJB2. This review focuses primarily on three alleles: $167 \Delta \mathrm{T}, 35 \Delta \mathrm{G}$, and $235 \Delta \mathrm{C}$. These alleles are recessive for nonsyndromic prelingual sensorineural hearing loss, and the evidence suggests complete penetrance but variable expressivity. Genet Med 2002:4(4):258-274.
\end{abstract}

Key Words: GJB2, connexin 26, hearing loss

Note: The term hearing loss is used in this article instead of the term hearing impairment, which is considered to be pejorative by people who are deaf or hard of hearing. ${ }^{1}$ We use the term hearing loss to include all levels of loss (mild to profound) and any age of onset including congenital losses. The word deaf refers to hearing status as determined by an audiogram. The word Deaf refers to the cultural community of people who are deaf and hard of hearing. $^{2}$

\section{GENE}

The Gap Junction Beta 2 or GJB2 gene (GenBank M86849, OMIM: $\left.{ }^{\star} 121011\right)$ resides at the chromosomal location $13 \mathrm{q} 11$ and encodes for the protein connexin 26 , a beta class gap junction protein expressed in the cochlea and in the epidermis. ${ }^{3}$ Connexin 26 hexamers form channels between cells that, when open, allow cell-to-cell diffusion of small molecules. This function is necessary for recycling potassium in the cochlea that plays a critical role in sensorineural hearing function. ${ }^{4}$ The GJB2 gene is small, with the entire coding region of 680 base pairs falling within exon 2 .

\section{GENE VARIANTS}

Aided by the relatively small size of the GJB2 gene, the flourish of activity on the genetics of hearing loss has resulted in rapid identification of GJB2 variants. Missense, nonsense, frameshift, insertion, and deletion variants have all been reported. To identify published genetic epidemiology studies re-

\footnotetext{
From the National Center on Birth Defects and Developmental Disabilities, Centers for Disease Control and Prevention, Atlanta, Georgia.

Aileen Kenneson, PhD, National Center on Birth Defects and Developmental Disabilities, 4770 Buford Highway NE, Mailstop F-15, Atlanta, GA 30341-3724.

Received: July 13, 2001.

Accepted: April 22, 2002.
}

DOI: 10.1097/01.GIM.0000020750.60733.CA lated to GJB2, we searched the MEDLINE database using the keywords GJB2, connexin 26, and hearing loss to identify relevant studies. References in related studies were also reviewed.

A list of GJB2 variants is presented in Table 1. Some variants are benign polymorphisms, with a high prevalence rate in various populations. For example, the V27I, E114G, and I203T variants were found on $54 \%, 55 \%$, and $94 \%$ of Japanese chromosomes, respectively. 5,6

The $167 \Delta \mathrm{T}, 35 \Delta \mathrm{G}$ (also known as $30 \Delta \mathrm{G}$ ), $235 \Delta \mathrm{C}$, and R143W alleles are the most common hearing loss-associated GJB2 alleles in the Ashkenazi Jewish, Caucasian, Japanese, and Ghanian populations, respectively. The best-characterized population is Caucasian of northern European descent. Table 2 presents the heterozygote carrier frequencies of the first three alleles either in the general population (hearing status unknown) or in control groups (without hearing loss). Ascertainment details were generally lacking and are listed in Table 2 as described in the publication. Likewise, descriptive information, including sex and age, were generally not provided. Despite these limitations, the studies consistently reported a prevalence of the $35 \Delta \mathrm{G}$ allele in the range of $1 \%$ to $3 \%$. In fact, one population-based study, which genotyped 560 randomly selected neonatal bloodspots in the Midwestern United States, detected a $35 \Delta G$ heterozygosity rate of $2.5 \%$ in this predominantly Caucasian population. ${ }^{7}$

In addition to Caucasians, published studies have focused on Mediterranean, Japanese, Korean, and Ashkenazi Jewish populations. The $35 \Delta \mathrm{G}$ allele is common in individuals of Mediterranean descent ( 1 in 30) and GJB2 testing has begun to be included in prenatal genetic counseling in Greece on a pilot basis. ${ }^{8}$ Although the numbers were small, and ascertainment and demographic details were generally lacking, studies indicated that $10 \%$ of Ashkenazi Jews carry the $167 \Delta \mathrm{T}$ allele, and $1 \%$ of Japanese and Korean individuals carry the $235 \Delta \mathrm{C}$ allele (Table 2).

The $35 \Delta \mathrm{G}, 167 \Delta \mathrm{T}$, and $235 \Delta \mathrm{C}$ alleles are all recessive alleles associated with nonsyndromic prelingual hearing loss. No ho- 
Table 1

Published GJB2 variants with corresponding change in the connexin 26 protein and the putative allele type

\begin{tabular}{|c|c|c|c|c|c|c|c|}
\hline Nucleotide change & Protein change & Putative allele type & Ref & Nucleotide change & Protein change & Putative allele type & Ref \\
\hline$-3712 \mathrm{G} \rightarrow \mathrm{A}$ & Splice site & & 7 & $293 \mathrm{G} \rightarrow \mathrm{A}$ & R98Q & Polymorphism & $\overline{7}$ \\
\hline$-3170 \mathrm{G} \rightarrow \mathrm{A}$ & Splice site & Recessive & 20 & $298 \mathrm{C} \rightarrow \mathrm{T}$ & H100Y & Polymorphism & 7 \\
\hline \multirow[t]{2}{*}{$1 \mathrm{~A} \rightarrow \mathrm{G}$} & M1V & Recessive & 44 & $299 \Delta \mathrm{AT}$ & Frameshift & Recessive & 34 \\
\hline & $\mathrm{T} 8 \mathrm{M}$ & Recessive & 30 & $304 \Delta \mathrm{GAG}$ & $\mathrm{E} 100 \Delta$ & Recessive & 75 \\
\hline $31 \Delta 14$ & Frameshift & & 43 & $312 \Delta 14$ & Frameshift & Recessive & 35 \\
\hline $31 \Delta 38$ & Frameshift & & 35 & $316 \Delta 14$ & Frameshift & Recessive & 49 \\
\hline $35 \mathrm{G} \rightarrow \mathrm{T}$ & $\mathrm{G} 12 \mathrm{~V}$ & Recessive & 70 & $333 \Delta \mathrm{AA}$ & Frameshift & Recessive & 49 \\
\hline $35 \Delta \mathrm{G}$ & Frameshift & Recessive & 71 & $339 \mathrm{~T} \rightarrow \mathrm{G}$ & S113R & Recessive & 49 \\
\hline 35 insG & Frameshift & Recessive & 44 & $341 \mathrm{~A} \rightarrow \mathrm{G}$ & E114G & Polymorphism & 5 \\
\hline $51 \Delta 12$ insA & Frameshift & Recessive & 24 & $358 \Delta \mathrm{GAG}$ & $\mathrm{E} 120 \Delta$ & Recessive & 20 \\
\hline $56 \mathrm{G} \rightarrow \mathrm{C}$ & $\mathrm{S} 19 \mathrm{~T}$ & Recessive & 70 & $365 \mathrm{~A} \rightarrow \mathrm{T}$ & K122I & Recessive & 7 \\
\hline $59 \mathrm{~T} \rightarrow \mathrm{C}$ & $\mathrm{I} 20 \mathrm{~T}$ & Recessive & 22 & $367 \mathrm{~A} \rightarrow \mathrm{G}$ & T123A & Polymorphism & 56 \\
\hline $71 \mathrm{G} \rightarrow \mathrm{A}$ & W24X & & 3 & & $\mathrm{~T} 123 \mathrm{~N}$ & & 41 \\
\hline $79 \mathrm{G} \rightarrow \mathrm{A}$ & V27I & Polymorphism & 49 & $370 \mathrm{C} \rightarrow \mathrm{T}$ & Q124X & Recessive & 74 \\
\hline \multirow[t]{2}{*}{$90 \mathrm{~T} \rightarrow \mathrm{A} / \mathrm{C}$} & I30I & Polymorphism & 71 & $380 \mathrm{G} \rightarrow \mathrm{A}$ & $\mathrm{R} 127 \mathrm{H}$ & Recessive & 44 \\
\hline & $\mathrm{R} 32 \mathrm{C}$ & Recessive & 53 & & R127C & & 41 \\
\hline $95 \mathrm{G} \rightarrow \mathrm{A}$ & $\mathrm{R} 32 \mathrm{H}$ & & 39 & $384 \mathrm{C} \rightarrow \mathrm{T}$ & I128I & Polymorphism & 71 \\
\hline \multirow[t]{2}{*}{$101 \mathrm{~T} \rightarrow \mathrm{C}$} & M34T & & 3 & & E129K & & 30 \\
\hline & I35S & & 41 & $408 \mathrm{C} \rightarrow \mathrm{A}$ & Y136X & Recessive & 5 \\
\hline $109 \mathrm{G} \rightarrow \mathrm{A}$ & V37I & & 49 & $416 \mathrm{G} \rightarrow \mathrm{A}$ & S139N & & 39 \\
\hline $122 \mathrm{~A} \rightarrow \mathrm{G}$ & K41R & Polymorphism & 38 & $427 \mathrm{C} \rightarrow \mathrm{T}$ & R143W & Recessive & 76 \\
\hline \multirow[t]{2}{*}{125 delAGG } & $\mathrm{E} 42 \Delta$ & Dominant and & 72 & $428 \mathrm{G} \rightarrow \mathrm{A}$ & R143Q & & 22 \\
\hline & & Vohwinkel syndrome & & & & & \\
\hline $132 \mathrm{G} \rightarrow \mathrm{A}$ & W44C & Dominant & 18 & $445 \mathrm{G} \rightarrow \mathrm{A}$ & A149T & & 70 \\
\hline $132 \mathrm{G} \rightarrow \mathrm{A}$ & W44X & Recessive & 7 & $457 \mathrm{G} \rightarrow \mathrm{A}$ & V153I & & 39 \\
\hline $134 \mathrm{G} \rightarrow \mathrm{A}$ & G45E & Recessive & 5,6 & $465 \mathrm{~T} \rightarrow \mathrm{A}$ & Y1555X & & 22 \\
\hline \multirow[t]{3}{*}{$139 \mathrm{G} \rightarrow \mathrm{T}$} & E47X & Recessive & 20 & $478 \mathrm{G} \rightarrow \mathrm{A}$ & G160S & Polymorphism & 74 \\
\hline & $\mathrm{E} 47 \mathrm{~K}$ & Recessive & 53 & $487 \mathrm{~A} \rightarrow \mathrm{G}$ & M163V & & 39 \\
\hline & C53R & & 41 & 504 insAACG & Frameshift & Recessive & 52 \\
\hline $167 \Delta \mathrm{T}$ & Frameshift & Recessive & 72 & 509 insA & Frameshift & Recessive & 20 \\
\hline $169 \mathrm{C} \rightarrow \mathrm{T}$ & Q57X & Recessive & 45 & $511 \mathrm{G} \rightarrow \mathrm{A}$ & & & 52 \\
\hline $176 \mathrm{C} \rightarrow \mathrm{G}$ & G59A & Dominant and & 55 & & P173R & Recessive & 70 \\
\hline & & Vohwinkel syndrome & & & & & \\
\hline $176 \Delta 16$ & Frameshift & Recessive & 5,6 & $523 \mathrm{C} \rightarrow \mathrm{T}$ & P175T & Recessive & 20 \\
\hline $195 \mathrm{C} \rightarrow \mathrm{G}$ & Y65X & Recessive & 44 & $533 \mathrm{~T} \rightarrow \mathrm{C}$ & V178T & Recessive & 25 \\
\hline \multirow[t]{2}{*}{$196 \mathrm{G} \rightarrow \mathrm{C}$} & $\mathrm{D} 66 \mathrm{H}$ & Dominant and & 54 & $546 \mathrm{G} \rightarrow \mathrm{C}$ & V182V & Polymorphism & 74 \\
\hline & & Vohwinkel syndrome & & & & & \\
\hline $215 \mathrm{C} \rightarrow \mathrm{G}$ & $\mathrm{S} 72 \mathrm{C}$ & Polymorphism & 38 & $551 \mathrm{G} \rightarrow \mathrm{C}$ & $\mathrm{R} 184 \mathrm{P}$ & Recessive & 35,43 \\
\hline \multirow[t]{2}{*}{$223 \mathrm{~T} \rightarrow \mathrm{G}$} & R75W & Dominant and & 56 & & R184Q & Dominant & 74 \\
\hline & & Vohwinkel syndrome & & & & & \\
\hline $229 \mathrm{~T} \rightarrow \mathrm{C}$ & W77R & & 73 & $589 \mathrm{G} \rightarrow \mathrm{T}$ & A197S & & 25 \\
\hline $231 \mathrm{G} \rightarrow \mathrm{A}$ & W77X & Recessive & 3 & $596 \mathrm{C} \rightarrow \mathrm{T}$ & S199F & Recessive & 7 \\
\hline $235 \Delta \mathrm{C}$ & Frameshift & Recessive & 5,6 & $605 \mathrm{G} \rightarrow \mathrm{T}$ & $\mathrm{C} 202 \mathrm{~F}$ & Dominant & 36 \\
\hline $236 \mathrm{~T} \rightarrow \mathrm{C}$ & L79P & Recessive & 25 & $608 \mathrm{TC} \rightarrow \mathrm{AA}$ & I203K & Recessive & 25 \\
\hline $249 \mathrm{C} \rightarrow \mathrm{G}$ & F83L & Polymorphism & 74 & $608 \mathrm{~T} \rightarrow \mathrm{C}$ & I203T & Polymorphism & 5,6 \\
\hline $250 \mathrm{G} \rightarrow \mathrm{C}$ & V84L & Recessive & 3 & $617 \mathrm{~A} \rightarrow \mathrm{G}$ & N206S & & 30,39 \\
\hline $251 \mathrm{~T} \rightarrow \mathrm{C}$ & V84A & Polymorphism & 38 & $631 \Delta \mathrm{GT}$ & Frameshift & Recessive & 49 \\
\hline $253 \mathrm{~T} \rightarrow \mathrm{C}$ & S85P & Recessive & 38 & $641 \mathrm{~T} \rightarrow \mathrm{C}$ & L214P & Recessive & 25 \\
\hline $267 \mathrm{C} \rightarrow \mathrm{A}$ & L89L & Polymorphism & 71 & $645 \Delta$ TAGA & Frameshift & Recessive & 53 \\
\hline $269 \mathrm{~T} \rightarrow \mathrm{C}$ & L90P & Recessive & 20,43 & $670 \mathrm{~A} \rightarrow \mathrm{C}$ & K224Q & Recessive & 77 \\
\hline 269 ins T & Frameshift & Recessive & 20 & & & & \\
\hline $283 \mathrm{G} \rightarrow \mathrm{A}$ & V95M & Recessive & 49 & & & & \\
\hline
\end{tabular}


Table 2

Heterozygote rates of three GJB2 mutations among control populations

\begin{tabular}{|c|c|c|c|c|c|c|c|}
\hline \multirow[b]{2}{*}{ Location } & \multirow[b]{2}{*}{ Ref } & \multirow[b]{2}{*}{ Description of control group ${ }^{a}$} & \multirow[b]{2}{*}{ DNA analysis method } & \multicolumn{3}{|c|}{$\begin{array}{l}\text { No. of heterozygotes, heterozygote } \\
\text { frequency mean, and } 95 \% \\
\text { confidence intervals }\end{array}$} & \multirow[b]{2}{*}{$N$} \\
\hline & & & & $35 \Delta \mathrm{G}$ & $167 \Delta \mathrm{T}$ & $235 \Delta \mathrm{C}$ & \\
\hline Australia & 41 & $\begin{array}{l}\text { Newborns (dried bloodspots) born in } \\
\text { Victoria in May } 1986\end{array}$ & $\begin{array}{l}\text { PCR-based; additional details } \\
\text { unspecified }\end{array}$ & $\begin{array}{c}10 \\
(1.0 \%) \\
(0.48-1.83)\end{array}$ & & & 1,000 \\
\hline Belgium & 78 & $\begin{array}{l}\text { Ascertainment not described; unrelated } \\
\text { unaffected }\end{array}$ & PCR with restriction analysis ${ }^{b}$ & $\begin{array}{c}9 \\
2.50 \% \\
(1.15-4.69)\end{array}$ & & & 360 \\
\hline Brazil & 79 & Randomly selected neonates & Allele-specific $\mathrm{PCR}^{b}$ & $\begin{array}{c}6 \\
0.97 \% \\
(0.36-2.09)\end{array}$ & & & 620 \\
\hline \multirow[t]{2}{*}{ Egypt } & 26 & $\begin{array}{l}\text { Ascertainment not described; unrelated } \\
\text { random; 1:1 male:female ratio }\end{array}$ & $\begin{array}{l}\text { PCR with restriction analysis or } \\
\text { allele-specific hybridization }{ }^{b}\end{array}$ & $\begin{array}{c}0 \\
0 \% \\
(0-3.81)\end{array}$ & & & 95 \\
\hline & 56 & $\begin{array}{l}\text { Individuals without skin disorders from } \\
\text { general genetics clinic, } 77 \text { Egyptians and } \\
17 \text { Caucasians }\end{array}$ & PCR with restriction analysis ${ }^{b}$ & $\begin{array}{c}1 \\
\text { (Egyptian) } \\
1.3 \% \\
(0.03-7.02)\end{array}$ & & & 94 \\
\hline Europe & 26 & $\begin{array}{l}\text { Ascertainment not described; unrelated } \\
\text { random; 1:1 male:female ratio }\end{array}$ & $\begin{array}{l}\text { PCR with restriction analysis or } \\
\text { allele-specific hybridization }\end{array}$ & $\begin{array}{c}64 \\
1.96 \% \\
(1.51-2.49)\end{array}$ & & & 3,270 \\
\hline \multirow[t]{2}{*}{ France } & 35 & $\begin{array}{l}68 \text { unrelated individuals (ascertainment } \\
\text { not described) and } 51 \mathrm{CEPH} \\
\text { individuals }\end{array}$ & Sequencing & $\begin{array}{c}0 \\
0 \% \\
(0-3.05)\end{array}$ & $\begin{array}{c}0 \\
0 \% \\
(0-3.05)\end{array}$ & $\begin{array}{c}0 \\
0 \% \\
(0-3.05)\end{array}$ & 119 \\
\hline & 80 & $\begin{array}{l}\text { Newborns (dried blood spots) born in } \\
1990-1991\end{array}$ & Allele-specific $\mathrm{PCR}^{b}$ & $\begin{array}{c}14 \\
2.73 \% \\
(1.50-4.55)\end{array}$ & & & 512 \\
\hline Greece & 81 & $\begin{array}{l}\text { Healthy blood donors aged } 18-60 \text { years } \\
\quad(\text { mean }=30.8)\end{array}$ & $\begin{array}{l}\text { Allele-specific PCR or PCR } \\
\text { with allele-specific } \\
\text { hybridization }^{b}\end{array}$ & $\begin{array}{c}14 \\
3.54 \% \\
(1.95-5.88)\end{array}$ & & & 395 \\
\hline \multirow[t]{3}{*}{$\begin{array}{l}\text { Israel__Ashkenazi } \\
\text { Jewish }\end{array}$} & 82 & Samples from genetics lab & Sequencing & $\begin{array}{c}1 \\
0.21 \% \\
(0.01-1.19)(2\end{array}$ & $\begin{array}{c}113 \\
24.2 \% \\
0.3-28.1)\end{array}$ & $\begin{array}{c}0 \\
0 \% \\
(0-0.79)\end{array}$ & 467 \\
\hline & 33 & $\begin{array}{l}\text { Individuals undergoing genetic testing for } \\
\text { carrier status for Tay-Sachs, etc. }\end{array}$ & PCR with restriction analysis ${ }^{b}$ & $\begin{array}{c}1 \\
1.09 \% \\
(0.03-5.91)\end{array}$ & & & 92 \\
\hline & & & & & $\begin{array}{c}20 \\
7.46 \% \\
2-11.29)\end{array}$ & & 268 \\
\hline Italy & 44 & $\begin{array}{l}\text { Ascertainment not described; unrelated } \\
\text { general population }\end{array}$ & $\begin{array}{l}\text { SSCP followed by sequencing if } \\
\text { positive }\end{array}$ & $\begin{array}{c}6 \\
4.0 \% \\
(1.48-8.50)\end{array}$ & $\begin{array}{c}0 \\
0 \% \\
(0-2.43)\end{array}$ & $\begin{array}{c}0 \\
0 \% \\
(0-2.43)\end{array}$ & 150 \\
\hline \multirow[t]{4}{*}{ Japan } & 34 & $\begin{array}{l}\text { Ascertainment not described; unrelated } \\
\text { individuals known not to have } \\
\text { noticeable hearing loss }\end{array}$ & Sequencing & $\begin{array}{c}0 \\
0 \% \\
(0-3.77)\end{array}$ & $\begin{array}{c}0 \\
0 \% \\
(0-3.77)(\end{array}$ & $\begin{array}{r}2 \\
2.08 \% \\
.25-7.32)\end{array}$ & 96 \\
\hline & 5 & Ascertainment not described & Sequencing & $\begin{array}{c}0 \\
0 \% \\
(0-7.11)\end{array}$ & $\begin{array}{c}0 \\
0 \% \\
(0-7.11)\end{array}$ & $\begin{array}{c}0 \\
0 \% \\
(0-7.11)\end{array}$ & 50 \\
\hline & 6 & $\begin{array}{l}\text { Ascertainment not described; unrelated } \\
\text { individuals with normal hearing; } 95 \\
\text { males, } 108 \text { females }\end{array}$ & Sequencing & $\begin{array}{c}0 \\
0 \% \\
(0-5.69)\end{array}$ & $\begin{array}{c}0 \\
0 \% \\
(0-5.69)\end{array}$ & & 63 \\
\hline & & & & & & $\begin{array}{c}2 \\
0.99 \% \\
.12-3.51)\end{array}$ & 203 \\
\hline Korea & 38 & $\begin{array}{l}\text { Blood samples from healthy newborns } \\
\text { with normal OAE }\end{array}$ & Sequencing & $\begin{array}{c}1 \\
1.00 \% \\
(0.02-5.45)\end{array}$ & $\begin{array}{c}0 \\
0 \% \\
(0-3.62)(\end{array}$ & $\begin{array}{c}1 \\
1.00 \% \\
.02-5.45)\end{array}$ & 100 \\
\hline $\begin{array}{l}\text { Middle } \\
\text { East_Jewish }\end{array}$ & 26 & $\begin{array}{l}\text { Ascertainment not described; unrelated } \\
\text { random; 1:1 male:female ratio }\end{array}$ & $\begin{array}{l}\text { PCR with restriction analysis or } \\
\text { allele-specific hybridization }\end{array}$ & $\begin{array}{c}5 \\
1.33 \% \\
(0.43-3.08)\end{array}$ & & & 376 \\
\hline Oman & 83 & Ascertainment not described & PCR with restriction analysis ${ }^{b}$ & $\begin{array}{c}0 \\
0 \% \\
(0-1.31)\end{array}$ & $\begin{array}{c}0 \\
0 \% \\
(0-1.31)\end{array}$ & & 280 \\
\hline
\end{tabular}


Table 2

Continued

\begin{tabular}{|c|c|c|c|c|c|c|c|}
\hline \multirow[b]{2}{*}{ Location } & \multirow[b]{2}{*}{ Ref } & \multirow[b]{2}{*}{ Description of control group ${ }^{a}$} & \multirow[b]{2}{*}{ DNA analysis method } & \multicolumn{3}{|c|}{$\begin{array}{l}\text { No. of heterozygotes, heterozygote } \\
\text { frequency mean, and } 95 \% \\
\text { confidence intervals }\end{array}$} & \multirow[b]{2}{*}{$N$} \\
\hline & & & & $35 \Delta \mathrm{G}$ & $167 \Delta \mathrm{T}$ & $235 \Delta \mathrm{C}$ & \\
\hline Poland & 84 & $\begin{array}{l}\text { Ascertainment not described; unrelated } \\
\text { random; 1:1 male:female ratio }\end{array}$ & $\begin{array}{l}\text { PCR with allele-specific } \\
\text { hybridization }^{b}\end{array}$ & $\begin{array}{c}3 \\
2.00 \% \\
(0.41-5.73)\end{array}$ & & & 150 \\
\hline Russia & 85 & $\begin{array}{l}\text { Ascertainment not described; five ethnic } \\
\text { groups: } 194 \text { Mari, 51 Komi, 154 } \\
\text { Chuvashs, } 106 \text { Yakuts, } 55 \text { Bashkirs }\end{array}$ & PCR with gel electrophoresis ${ }^{b}$ & $\begin{array}{c}12 \\
2.14 \% \\
(1.11-3.71)\end{array}$ & & & 560 \\
\hline Spain & 44 & $\begin{array}{l}\text { Ascertainment not described; unrelated } \\
\text { general population }\end{array}$ & Sequencing & $\begin{array}{c}3 \\
2.31 \% \\
(0.48-6.60)\end{array}$ & $\begin{array}{c}0 \\
0 \\
(0-2.8)\end{array}$ & $\begin{array}{c}0 \\
0-2.8)\end{array}$ & 130 \\
\hline Tunisia & 86 & $\begin{array}{l}\text { Ascertainment not described; unrelated } \\
\text { general population }\end{array}$ & DGGE & $\begin{array}{c}3 \\
1.27 \% \\
(0.26-3.67)\end{array}$ & & & 236 \\
\hline Turkey & 87 & $\begin{array}{l}\text { Individuals from unrelated research } \\
\text { projects with no known hearing loss }\end{array}$ & PCR with restriction analysis ${ }^{b}$ & $\begin{array}{c}12 \\
1.78 \% \\
(0.92-3.09)\end{array}$ & $\begin{array}{c}0 \\
0 \% \\
(0-0.55)\end{array}$ & & 674 \\
\hline \multirow[t]{3}{*}{$\begin{array}{l}\text { U.S.A.-African } \\
\text { American }\end{array}$} & 26 & $\begin{array}{l}\text { Ascertainment not described; unrelated } \\
\text { random; 1:1 male:female ratio }\end{array}$ & $\begin{array}{l}\text { PCR with restriction analysis or } \\
\text { allele-specific hybridization }{ }^{b}\end{array}$ & $\begin{array}{c}0 \\
0 \% \\
(0-1.92)\end{array}$ & & & 190 \\
\hline & 28 & $\begin{array}{l}\text { Samples collected at Clinical Genetics } \\
\text { Section of Michigan State University } \\
\text { from individuals seeking counseling for } \\
\text { disorders other than hearing loss }\end{array}$ & $\begin{array}{l}\text { SSCP followed by sequencing if } \\
\text { positive }\end{array}$ & $\begin{array}{c}0 \\
0 \% \\
(0-2.11)\end{array}$ & & & 173 \\
\hline & & & & & $\begin{array}{c}0 \\
0 \% \\
(0-2.13)\end{array}$ & & 171 \\
\hline \multirow[t]{3}{*}{$\begin{array}{l}\text { U.S.A.-Ashkenazi } \\
\text { Jewish }\end{array}$} & 28 & $\begin{array}{l}\text { Individuals undergoing genetic testing for } \\
\text { carrier status for Tay-Sachs, etc. }\end{array}$ & $\begin{array}{l}\text { SSCP followed by sequencing if } \\
\text { positive }\end{array}$ & $\begin{array}{c}4 \\
0.78 \% \\
(0.21-1.99)\end{array}$ & & & 551 \\
\hline & & & & & $\begin{array}{r}22 \\
4.03 \% \\
2.54-6.04)\end{array}$ & & 546 \\
\hline & 88 & & $\begin{array}{l}\text { PCR with allele-specific } \\
\text { hybridization }^{b}\end{array}$ & $\begin{array}{c}7 \\
0.69 \% \\
(0.28-1.42)(\end{array}$ & $\begin{array}{r}40 \\
3.95 \% \\
2.84-5.34)\end{array}$ & & 1,012 \\
\hline U.S.A.—Asian & 28 & $\begin{array}{l}\text { Samples collected at Clinical Genetics } \\
\text { Section of Michigan State University } \\
\text { from individuals seeking counseling for } \\
\text { disorders other than hearing loss }\end{array}$ & $\begin{array}{l}\text { SSCP followed by sequencing if } \\
\text { positive }\end{array}$ & $\begin{array}{c}0 \\
0 \% \\
(0-6.85)\end{array}$ & $\begin{array}{c}0 \\
0 \% \\
(0-6.85)\end{array}$ & & 52 \\
\hline U.S.A.—Caucasian & 28 & $\begin{array}{l}\text { Samples collected at Clinical Genetics } \\
\text { Section of Michigan State University } \\
\text { from individuals seeking counseling for } \\
\text { disorders other than hearing loss }\end{array}$ & $\begin{array}{l}\text { SSCP followed by sequencing if } \\
\text { positive }\end{array}$ & $\begin{array}{c}1 \\
0.58 \% \\
(0.01-3.18)\end{array}$ & $\begin{array}{c}0 \\
0 \% \\
(0-2.09)\end{array}$ & & $\begin{array}{l}173 \\
175\end{array}$ \\
\hline \multirow[t]{3}{*}{$\begin{array}{l}\text { U.S.A.-Midwest } \\
\text { (primarily } \\
\text { Caucasian) }\end{array}$} & 7 & $\begin{array}{l}\text { Randomly selected neonatal bloodspots: } \\
94.8 \% \text { non-Hispanic white, } 1.9 \% \text { black, } \\
1.7 \% \text { Hispanic, } 1.3 \% \text { Asian and Pacific } \\
\text { Islander, } 0.3 \% \text { American Indian, } 0.2 \% \\
\text { Ashkenazi Jewish }\end{array}$ & Allele-specific $\mathrm{PCR}^{b}$ & $\begin{array}{c}14 \\
2.5 \% \\
(1.37-4.16)\end{array}$ & & & 560 \\
\hline & 49 & $\begin{array}{l}\text { Unrelated individuals known not to have } \\
\text { noticeable hearing loss, self-assessed as } \\
\text { primarily Caucasian of Northern and } \\
\text { Southern European origin. No Asian, } \\
\text { African or Native Americans were } \\
\text { available }\end{array}$ & $\begin{array}{l}\text { Heteroduplex analysis followed } \\
\text { by sequencing if positive }\end{array}$ & $\begin{array}{c}2 \\
2.08 \% \\
(0.25-7.32)\end{array}$ & $\begin{array}{c}0 \\
0 \% \\
(0-3.77)\end{array}$ & & 96 \\
\hline & 74 & $\begin{array}{l}\text { Ascertainment not described; random } \\
\text { individuals }\end{array}$ & $\begin{array}{l}\text { Allele-specific PCR and SSCP } \\
\text { followed by sequencing if } \\
\text { positive }\end{array}$ & $\begin{array}{c}1 \\
1.00 \% \\
(0.02-5.45)\end{array}$ & & & 100 \\
\hline
\end{tabular}

SSCP, single-strand conformation polymorphism; OAE, otoacoustic emission; DGGE, denaturing gradient gel electrophoresis; CEPH, Centre Etude Polymorphisme Humaine.

${ }^{a}$ Age and sex unspecified unless noted otherwise.

${ }^{b}$ Method does not distinguish between heterozygotes and compound heterozygotes. 
mozygotes for any of these three alleles have been reported in control groups. Some of the methods used tested only for specific alleles; therefore, distinguishing between heterozygotes and compound heterozygotes is not possible. This limitation affects the conclusions that can be drawn regarding penetrance in these studies. However, in the studies that did fully characterize the second allele in the control groups, no compound heterozygotes were reported. This finding suggests that for these three common alleles, i.e., $167 \Delta \mathrm{T}, 35 \Delta \mathrm{G}$, and $235 \Delta \mathrm{C}$, penetrance of hearing loss in homozygotes is complete. However, larger population-based studies are needed to support this model and to characterize the penetrance of the less common alleles.

\section{HEARING LOSS}

"With over a million essential moving parts, the auditory receptor organ, or cochlea, is the most complex mechanical apparatus in the human body." "Given this complexity, it is not surprising that sequence variation in any one of hundreds of genes can lead to hearing loss. Hearing loss occurs in approximately 1 to 3 of 1,000 children, ${ }^{10}$ and is generally attributed to pure genetic factors in approximately $50 \%$ of cases. ${ }^{11}$ In approximately $30 \%$ of cases, a specific syndrome can be identified, with more than 400 syndromes claiming hearing loss as a component. The other $70 \%$ of cases are nonsyndromic. ${ }^{11,12}$ Nonsyndromic cases may be familial or sporadic. The nature of familial nonsyndromic prelingual hearing loss is usually described as follows: $75 \%$ to $80 \%$ are autosomal recessive (designated with the prefix DFNB), $20 \%$ to $25 \%$ are autosomal dominant (DFNA), and $1 \%$ to $1.5 \%$ are $\mathrm{X}$-linked (DFN). ${ }^{13}$ The extraordinary genetic heterogeneity of hearing loss has been demonstrated by linkage studies, which indicated many loci for nonsyndromic hearing loss: 30 autosomal recessive, 29 autosomal dominant, and $8 \mathrm{X}$-linked. ${ }^{14}$ Two mitochondrial variants, A1555G and A7445G, also have been implicated in nonsyndromic hearing loss. Several other mitochondrial mutations are associated with syndromic forms of hearing loss. ${ }^{15}$ One of the autosomal recessive loci, DFNB1, was mapped to chromosome $13 \mathrm{q} 12^{16}$ and was attributed recently to the GJB2 gene. ${ }^{3}$ Although the majority of GJB2 variants are recessive, dominant alleles have been identified that account for the DFNA3 locus mapped to the same region. ${ }^{17,18}$

\section{ASSOCIATIONS}

\section{Contribution of GJB2 to hearing loss}

Given the extraordinary genetic heterogeneity of nonsyndromic hearing loss, it was surprising to find that sequence variations at the GJB2 locus account for up to $50 \%$ of cases of nonsyndromic prelingual sensorineural hearing loss in some populations. A recent model to explain this observation is based on the tradition of intermarriage between individuals with hearing loss in some populations. A gradual increase in the proportion of hearing loss due to a hypothetical autosomal recessive mutation would be a consequence of this assortative mating. ${ }^{19}$

More than 90 variants of the GJB2 gene have been reported, and many are rare. One variant generally predominates in any given population, such as $167 \Delta \mathrm{T}$ in the Ashkenazi Jewish population, $35 \Delta \mathrm{G}$ among Caucasians of northern European descent, $235 \Delta \mathrm{C}$ in the Japanese population, and R143W in Ghana. Table 3 summarizes studies of the prevalence of various GJB2 genotypes among individuals with prelingual hearing loss. The studies presented in this table vary in their ascertainment methods, case definitions, inclusion of presumably acquired cases, and genotyping methods. Because the genotype information that can be gleaned from the studies depends on the methodology, the data must be considered accordingly.

Twenty-two of the 30 studies in Table 3 used DNA sequence analysis to fully characterize both alleles in each individual. These studies provided the best estimate of the proportion of hearing loss cases associated with GJB2 variants. The percentages of cases of prelingual hearing loss associated with two variants in GJB2 (i.e., homozygotes or compound heterozygotes) for these studies were calculated (Table 3). Sequence variations at the GJB2 locus were detected in approximately $20 \%$ of individuals with nonsyndromic prelingual sensorineural hearing loss in populations of Caucasians of northern European descent. GJB2 variants were detected in approximately $5 \%$ of individuals with hearing loss in Korea, $14 \%$ in Australia, $17 \%$ in Tunisia, $20 \%$ in Japan, and $43 \%$ in Israel. Thus the contribution of GJB2 variants to hearing loss varied between populations. Table 3 also demonstrates that the frequencies of the various GJB2 alleles differed between the populations.

Most of these DNA-sequencing studies analyzed exon 2 of GJB2, which contains the entire coding region and 92 of the 94 variants described in Table 1. However, seven of the studies also sequenced exon 1, which contains the 3' untranslated region and the other two known variants. ${ }^{7,20-25}$ Only one of these studies detected a sequence variation in exon 1,20 suggesting that exon 2 analysis will detect most of the mutations in GJB2 in individuals with hearing loss. However, studies with larger groups are necessary to determine the frequency of the variants found in exon 1.

Other studies used methods that detect only specific alleles, or reported data only on the major alleles, particularly $35 \Delta \mathrm{G}$. Because these data did not fully characterize the second allele, heterozygotes could not be distinguished from compound heterozygotes (Table 3 ). These studies also tended to include small numbers and generally lacked ascertainment and descriptive details. However, despite these limitations, the study results were consistent with those presented above and indicated that the $35 \Delta \mathrm{G}$ allele accounts for approximately $10 \%$ to $20 \%$ of cases of hearing loss in Caucasians of northern European descent, but approximately $30 \%$ to $40 \%$ of cases in Mediterranean regions. 
Table 3

Contribution of GJB2 variants to hearing loss: Summary of GJB2 genotypes characterized in cases of hearing loss (familial, non-familial, ${ }^{a}$ and both)

\begin{tabular}{|c|c|c|c|c|c|c|}
\hline$\frac{\text { Location }}{\text { Australia }}$ & \multirow{2}{*}{$\frac{\text { Ref }}{40}$} & \multicolumn{2}{|l|}{ Case definition \& methods ${ }^{b}$} & \multicolumn{2}{|c|}{ Genotypes present } & \multirow{2}{*}{$\begin{array}{l}\begin{array}{l}\% \text { of cases with two GJB2 } \\
\text { variant alleles }\end{array} \text { (95\% CI) } \\
13.5 \% \\
(6.6-23.4)\end{array}$} \\
\hline Australia & & $\begin{array}{l}\text { Nonsyndromic sensorineural mild to } \\
\text { profound, unilateral or bilateral, } \\
\text { hearing loss patients at a Pediatric } \\
\text { Hearing Loss Investigation Clinic, } \\
\text { excluding cases of known } \\
\text { environmental exposure and cases of } \\
\text { inner ear malformations, mean age } \\
=7 \text { years; sequenced exon } 2\end{array}$ & $\begin{array}{l}\text { Mixed } \\
N=74\end{array}$ & $\begin{array}{l}335 \Delta \mathrm{G} / 35 \Delta \mathrm{G} \\
135 \Delta \mathrm{G} / \mathrm{V} 371 \\
135 \Delta \mathrm{G} / 167 \Delta \mathrm{T} \\
1 \mathrm{M} 34 \mathrm{~T} / \mathrm{R} 184 \mathrm{~W} \\
3 \mathrm{M} 34 \mathrm{~T} /+\end{array}$ & $\begin{array}{l}235 \Delta \mathrm{G} / \mathrm{L} 90 \mathrm{P} \\
135 \Delta \mathrm{G} / \mathrm{M} 34 \mathrm{~T} \\
1 \mathrm{~V} 37 \mathrm{I} / \mathrm{V} 37 \mathrm{I} \\
435 \Delta \mathrm{G} /+ \\
1 \mathrm{~V} 37 \mathrm{I} /+\end{array}$ & \\
\hline & 41 & $\begin{array}{l}\text { Children unilateral or bilateral } \\
\text { nonsyndromic prelingual hearing } \\
\text { loss seen at clinics in Melbourne and } \\
\text { Sydney between January 1, 1998, and } \\
\text { October 31, 2000; age } 4 \text { weeks to } 16 \\
\text { years (median = } 4 \text { years); sequenced } \\
\text { exon 2 }\end{array}$ & $\begin{array}{l}\text { Mixed } \\
N=243\end{array}$ & $\begin{array}{l}1635 \Delta \mathrm{G} / 35 \Delta \mathrm{G} \\
4 \mathrm{~V} 37 \mathrm{I} /+ \\
235 \Delta \mathrm{G} / \mathrm{L} 90 \mathrm{P} \\
235 \Delta \mathrm{G} / \mathrm{M} 34 \mathrm{~T} \\
235 \Delta \mathrm{G} / \mathrm{W} 24 \mathrm{X} \\
135 \Delta \mathrm{G} / \mathrm{W} 77 \mathrm{R} \\
135 \Delta \mathrm{G} / \mathrm{R} 127 \mathrm{C} \\
1 \mathrm{M} 34 \mathrm{~T} / \mathrm{R} 184 \mathrm{~W} \\
1 \mathrm{~T} 123 \mathrm{~N} / \mathrm{T} 123 \mathrm{~N}\end{array}$ & $\begin{array}{l}1035 \Delta \mathrm{G} /+ \\
3 \mathrm{M} 34 \mathrm{~T} /+ \\
235 \Delta \mathrm{G} / \mathrm{V} 37 \mathrm{I} \\
235 \Delta \mathrm{G} / \mathrm{C} 53 \mathrm{R} \\
135 \Delta \mathrm{G} / 167 \Delta \mathrm{T} \\
135 \Delta \mathrm{G} / \mathrm{R} 143 \mathrm{~W} \\
1 \mathrm{~V} 37 \mathrm{I} / \mathrm{V} 37 \mathrm{I} \\
1 \mathrm{~W} 24 \mathrm{X} / \mathrm{I} 35 \mathrm{~S} \\
1333 \Delta \mathrm{AA} /+\end{array}$ & $\begin{array}{l}14.0 \% \\
(10.0-19.0)\end{array}$ \\
\hline \multirow[t]{2}{*}{ Austria } & 22 & $\begin{array}{l}\text { Sequential unrelated patients with } \\
\text { nonsyndromic sensorineural hearing } \\
\text { loss referred to centers for Hearing, } \\
\text { Speech, and Voice Disorders in } \\
\text { Austria; sequenced exons } 1 \text { \& } 2\end{array}$ & $\begin{array}{l}\text { Familial } \\
N=25\end{array}$ & $\begin{array}{l}435 \Delta \mathrm{G} / 35 \Delta \mathrm{G} \\
1 \mathrm{~L} 90 \mathrm{P} / \mathrm{R} 143 \mathrm{Q} \\
2 \mathrm{G} 160 \mathrm{~S} /+\end{array}$ & $\begin{array}{l}235 \Delta \mathrm{G} / \mathrm{L} 90 \mathrm{P} \\
135 \Delta \mathrm{G} /+\end{array}$ & $\begin{array}{l}28.0 \% \\
(10.4-45.6)\end{array}$ \\
\hline & & & $\begin{array}{l}\text { Non-familial } \\
N=44\end{array}$ & $\begin{array}{l}335 \Delta \mathrm{G} / 35 \Delta \mathrm{G} \\
1 \mathrm{~L} 90 \mathrm{P} / 314 \Delta 14 \\
1 \mathrm{~L} 90 \mathrm{P} /+\end{array}$ & $\begin{array}{l}135 \Delta \mathrm{G} / \mathrm{V} 84 \mathrm{~L} \\
1 \mathrm{~L} 90 \mathrm{P} / \mathrm{I} 20 \mathrm{~T} \\
135 \Delta \mathrm{G} /+\end{array}$ & $\begin{array}{l}13.6 \% \\
(5.2-27.4)\end{array}$ \\
\hline $\begin{array}{l}\text { Belgium \& United } \\
\text { Kingdom }\end{array}$ & 89 & $\begin{array}{l}\text { Non-consanguineous nonsyndromic } \\
\text { sensorineural hearing cases, } \\
\text { excluding acquired cases; allele- } \\
\text { specific PCR, followed by sequencing }\end{array}$ & $\begin{array}{l}\text { Mixed } \\
N=68\end{array}$ & $\begin{array}{l}335 \Delta \mathrm{G} / 35 \Delta \mathrm{G} \\
335 \Delta \mathrm{G} / \text { unknown } \\
62 \text { unknown/unknown }\end{array}$ & & N/A \\
\hline \multirow[t]{2}{*}{ France } & $20^{d}$ & $\begin{array}{l}\text { Nonsyndromic sensorineural hearing } \\
\text { loss patients recruited from } \\
\text { consecutive individuals attending } \\
\text { the genetic counseling service for } \\
\text { deaf people at two Parisian hospitals } \\
\text { between April } 1997 \text { and September } \\
\text { 1998; aged } 4 \text { to } 20 \text { years; } 73 \text { females } \\
\text { and } 67 \text { males; sequenced exons } 1 \& 2\end{array}$ & $\begin{array}{l}\text { Recessive }^{e} \\
N=39 \\
\text { Non-familial } \\
N=49\end{array}$ & $\begin{array}{l}1035 \Delta \mathrm{G} / 35 \Delta \mathrm{G} \\
135 \Delta \mathrm{G} /-3170 \mathrm{G} \rightarrow \mathrm{A} \\
135 \Delta \mathrm{G} / 312 \Delta 14 \\
535 \Delta \mathrm{G} /+\end{array}$ & $\begin{array}{l}135 \Delta \mathrm{G} / 333 \Delta \mathrm{AA} \\
135 \Delta \mathrm{G} / 509 \text { insA } \\
135 \Delta \mathrm{G} / \mathrm{P} 175 \mathrm{~T} \\
1 \mathrm{~L} 90 \mathrm{P} /+\end{array}$ & $\begin{array}{l}28.6 \% \\
(34.8-67.6)\end{array}$ \\
\hline & $90^{d}$ & $\begin{array}{l}\text { Individuals with sensorineural hearing } \\
\text { loss recruited from the genetic } \\
\text { counseling service for individuals } \\
\text { with hearing loss at the Pasteur } \\
\text { Hospital and at the Armaud- } \\
\text { Trousseau Children's Hospital, } \\
\text { Paris, April 1997-September 1998; } \\
\text { aged } 4 \text { years or greater; PCR with } \\
\text { allele-specific hybridization }\end{array}$ & $\begin{array}{l}\text { Mixed } \\
N=181\end{array}$ & $\begin{array}{l}5035 \Delta \mathrm{G} / 35 \Delta \mathrm{F} \\
4735 \Delta \mathrm{G} / \mathrm{unknown} \\
84 \text { unknown/unknown }\end{array}$ & & N/A \\
\hline $\begin{array}{l}\text { France \& United } \\
\quad \text { Kingdom }\end{array}$ & 35 & $\begin{array}{l}\text { Prelingual hearing loss; sequenced exon } \\
\quad 2\end{array}$ & $\begin{array}{l}\text { Familial (at } \\
\text { least one } \\
\text { affected } \\
\text { sibling) } \\
N=47\end{array}$ & $\begin{array}{l}835 \Delta \mathrm{G} / 35 \Delta \mathrm{G} \\
135 \Delta \mathrm{G} / 310 \Delta 14\end{array}$ & $1435 \Delta \mathrm{G} /+$ & $\begin{array}{l}19.2 \% \\
(9.2-33.3)\end{array}$ \\
\hline Germany & 47 & $\begin{array}{l}\text { Individuals with symmetric moderate } \\
\text { to profound hearing loss of } \\
\text { unknown cause; } 47 \text { under age } 12, \\
117 \text { over age } 12 \text {; PCR with allele- } \\
\text { specific hybridization }\end{array}$ & $\begin{array}{l}\text { Mixed } \\
N=164\end{array}$ & $\begin{array}{l}435 \Delta \mathrm{G} / 35 \Delta \mathrm{G} \\
935 \Delta \mathrm{G} / \text { unknown } \\
151 \text { unknown/unknown }\end{array}$ & & N/A \\
\hline Ghana & 25 & $\begin{array}{l}\text { Unrelated students with nonsyndromic } \\
\text { profound sensorineural hearing loss } \\
\text { at schools for the deaf in Ghana; } \\
\text { individuals with known } \\
\text { environmental risk factors were } \\
\text { excluded; aged } 6 \text { to } 20 \text { years; } \\
\text { sequenced exons } 1 \text { \& } 2\end{array}$ & $\begin{array}{l}\text { Unspecified } \\
N=365\end{array}$ & $\begin{array}{l}51 \mathrm{R} 143 \mathrm{~W} / \mathrm{R} 143 \mathrm{~W} \\
1 \mathrm{R} 143 \mathrm{~W} / 35 \mathrm{ins} \\
1 \mathrm{R} 143 \mathrm{~W} / \mathrm{I} 203 \mathrm{~K} \\
1 \mathrm{~V} 178 \mathrm{~A} / \mathrm{V} 178 \mathrm{~A} \\
1 \mathrm{~A} 197 \mathrm{~S} /+\end{array}$ & $\begin{array}{l}4 \mathrm{R} 143 \mathrm{~W} /+ \\
1 \mathrm{R} 143 \mathrm{~W} / \mathrm{L} 90 \mathrm{P} \\
1 \mathrm{R} 143 \mathrm{~W} / \mathrm{L} 214 \mathrm{P} \\
1 \mathrm{R} 184 \mathrm{Q} /+\end{array}$ & $\begin{array}{l}16.7 \% \\
(13.0-20.9)\end{array}$ \\
\hline
\end{tabular}


Table 3

(Continued)

\begin{tabular}{|c|c|c|c|c|c|c|}
\hline Location & Ref & Case definition \& methods ${ }^{b}$ & & \multicolumn{2}{|c|}{ Genotypes present } & $\begin{array}{l}\% \text { of cases with two GJB2 } \\
\text { variant alleles }^{c}(95 \% \mathrm{CI})\end{array}$ \\
\hline \multirow[t]{2}{*}{ Greece } & 77 & $\begin{array}{l}\text { Unrelated individuals with prelingual } \\
\text { nonsyndromic sensorineural hearing } \\
\text { loss who attended major referral } \\
\text { centers for prelingual hearing loss in } \\
\text { Greece; allele-specific PCR, DGGE, } \\
\text { and sequencing }\end{array}$ & $\begin{array}{l}\text { Familial } \\
\quad \text { (definition } \\
\quad \text { not } \\
\quad \text { reported) } \\
N=8\end{array}$ & $\begin{array}{l}235 \Delta \mathrm{G} / 35 \Delta \mathrm{G} \\
435 \Delta \mathrm{G} / \text { unknown } \\
2 \text { unknown/unknown }\end{array}$ & & N/A \\
\hline & & & $\begin{array}{l}\text { Non-familial } \\
N=18\end{array}$ & $\begin{array}{l}435 \Delta \mathrm{G} / 35 \Delta \mathrm{G} \\
1135 \Delta \mathrm{G} / \text { unknown } \\
3 \text { unknown/unknown }\end{array}$ & & N/A \\
\hline \multirow[t]{4}{*}{ Israel } & 33 & $\begin{array}{l}\text { Nonsyndromic prelingual mild to } \\
\text { profound hearing loss cases } \\
\text { attending a Speech and Hearing } \\
\text { Clinic, excluding cases of known } \\
\text { environmental factors; sequenced } \\
\text { exon } 2\end{array}$ & $\begin{array}{l}\text { Non-familial } \\
N=9\end{array}$ & $\begin{array}{l}4167 \Delta \mathrm{T} / 167 \Delta \mathrm{T} \\
1167 \Delta \mathrm{T} /+\end{array}$ & & $\begin{array}{l}44.4 \% \\
(13.7-78.8)\end{array}$ \\
\hline & & & $\begin{array}{l}\text { Recessive }^{e} \\
N=18\end{array}$ & $\begin{array}{l}8167 \Delta \mathrm{T} / 167 \Delta \mathrm{T} \\
1167 \Delta \mathrm{T} /+ \\
135 \Delta \mathrm{G} /+\end{array}$ & $\begin{array}{l}5167 \Delta \mathrm{T} / 35 \Delta \mathrm{G} \\
235 \\
\mathrm{G} / 35 \\
\mathrm{G}\end{array}$ & $\begin{array}{l}83.3 \% \\
(58.6-96.4)\end{array}$ \\
\hline & 24 & $\begin{array}{l}\text { Nonsyndromic prelingual mild to } \\
\text { profound sensorineural hearing loss, } \\
\text { excluding cases of infection, trauma, } \\
\text { acoustic trauma, ototoxic drug use, } \\
\text { rubella, or premature birth; } \\
\text { sequenced exons } 1 \text { \& } 2\end{array}$ & $\begin{array}{l}\text { Non-familial } \\
N=29\end{array}$ & $\begin{array}{l}335 \Delta \mathrm{G} / 35 \Delta \mathrm{G} \\
4167 \Delta \mathrm{T} / 167 \Delta \mathrm{T}\end{array}$ & $\begin{array}{l}235 \Delta \mathrm{G} / 167 \Delta \mathrm{T} \\
135 \Delta \mathrm{G} /+\end{array}$ & $\begin{array}{l}31.0 \% \\
(15.3-50.8)\end{array}$ \\
\hline & & & $\begin{array}{l}\text { Recessive }^{e} \\
N=46\end{array}$ & $\begin{array}{l}835 \Delta \mathrm{G} / 35 \Delta \mathrm{G} \\
4167 \Delta \mathrm{T} / 167 \Delta \mathrm{T} \\
151 \Delta \mathrm{Tins} / 51 \Delta \mathrm{Tins}\end{array}$ & $\begin{array}{l}335 \Delta \mathrm{G} / 167 \Delta \mathrm{T} \\
335 \Delta \mathrm{G} /+\end{array}$ & $\begin{array}{l}34.8 \% \\
(21.4-50.2)\end{array}$ \\
\hline \multirow[t]{5}{*}{ Italy } & 91 & $\begin{array}{l}\text { Consecutive unselected children with } \\
\text { nonsyndromic sensorineural hearing } \\
\text { loss from an audiology service who } \\
\text { were aged } 1 \text { to } 6 \text { years at onset; PCR } \\
\text { with allele-specific restriction } \\
\text { analysis }\end{array}$ & $\begin{array}{l}\text { Mixed } \\
N=90\end{array}$ & $\begin{array}{l}735 \Delta \mathrm{G} / 35 \Delta \mathrm{G} \\
3635 \Delta \mathrm{G} / \text { unknown } \\
47 \text { unknown/unknown }\end{array}$ & & N/A \\
\hline & 23 & $\begin{array}{l}\text { Nonsyndromic prelingual hearing loss } \\
\text { greater than } 40 \mathrm{~dB} \text { from audiology } \\
\text { and phoniatrics clinics, excluding } \\
\text { individuals with known risk factors; } \\
\text { SSCP and CSGE of exons } 1 \text { and } 2 \text {, } \\
\text { followed by sequencing if positive }\end{array}$ & $\begin{array}{l}\text { Mixed } \\
N=94\end{array}$ & $\begin{array}{l}2735 \Delta \mathrm{G} / 35 \Delta \mathrm{G} \\
335 \Delta \mathrm{G} / \mathrm{IVS}+1 \mathrm{G} \rightarrow \mathrm{A} \\
135 \Delta \mathrm{G} / 31 \Delta 14 \\
1118 \Delta \mathrm{E} / 167 \Delta \mathrm{T} \\
1 \mathrm{R} 184 \mathrm{P} /+ \\
2 \mathrm{M} 34 \mathrm{~T} /+\end{array}$ & $\begin{array}{l}535 \Delta \mathrm{G} / \mathrm{Q} 47 \mathrm{X} \\
235 \Delta \mathrm{G} / \mathrm{R} 184 \mathrm{P} \\
135 \Delta \mathrm{G} / \mathrm{L} 90 \mathrm{P} \\
335 \Delta \mathrm{G} /+ \\
131 \Delta 14 /+\end{array}$ & $\begin{array}{l}41.5 \% \\
(31.4-52.1)\end{array}$ \\
\hline & 43 & $\begin{array}{l}\text { Nonsyndromic prelingual } \\
\text { sensorineural hearing loss greater } \\
\text { than } 40 \mathrm{~dB} \text {; aged } 3 \text { to } 35 \text { years, mean } \\
=12 \text { years; } 21 \text { females and } 32 \text { males; } \\
\text { sequenced exon } 2\end{array}$ & $\begin{array}{l}\text { Non-familial } \\
N=25\end{array}$ & $\begin{array}{l}835 \Delta \mathrm{G} / 35 \Delta \mathrm{G} \\
1167 \Delta \mathrm{T} / \mathrm{E} 120 \Delta \\
1 \mathrm{R} 184 \mathrm{P} /+\end{array}$ & $\begin{array}{l}235 \Delta \mathrm{G} / \mathrm{E} 47 \mathrm{X} \\
335 \Delta \mathrm{G} /+\end{array}$ & $\begin{array}{l}44.0 \% \\
(24.4-65.1)\end{array}$ \\
\hline & & & $\begin{array}{l}\text { Recessive }^{e} \\
N=17\end{array}$ & $\begin{array}{l}335 \Delta \mathrm{G} / 35 \Delta \mathrm{G} \\
135 \Delta \mathrm{G} /+\end{array}$ & $\begin{array}{l}135 \Delta \mathrm{G} / \mathrm{E} 47 \mathrm{X} \\
1 \mathrm{~L} 90 \mathrm{P} /+\end{array}$ & $\begin{array}{l}23.5 \% \\
(6.8-49.9)\end{array}$ \\
\hline & & & $\begin{array}{l}\text { Familial (at } \\
\text { least one } \\
\text { affected } \\
\text { nonsibling } \\
\text { relative) } \\
N=11\end{array}$ & $\begin{array}{l}535 \Delta \mathrm{G} / 35 \Delta \mathrm{G} \\
1314 \Delta 14 /+\end{array}$ & $135 \Delta \mathrm{G} / \mathrm{L} 90 \mathrm{P}$ & $\begin{array}{l}54.6 \% \\
(23.4-83.2)\end{array}$ \\
\hline Italy \& Spain & 44 & $\begin{array}{l}\text { Nonsyndromic prelingual moderate to } \\
\text { profound hearing loss, includes cases } \\
\text { of infection, oto-trauma, and } \\
\text { ototoxic drug use; sequenced exon } 2\end{array}$ & $\begin{array}{l}\text { Mixed } \\
N=92\end{array}$ & $\begin{array}{l}2535 \Delta \mathrm{G} / 35 \Delta \mathrm{G} \\
235 \Delta \mathrm{G} /+\end{array}$ & $935 \Delta \mathrm{G} /$ other & $\begin{array}{l}37.0 \% \\
(27.1-47.7)\end{array}$ \\
\hline \multirow[t]{3}{*}{ Japan } & 35 & $\begin{array}{l}\text { Nonsyndromic bilateral mild to } \\
\text { profound sensorineural hearing loss } \\
\text { with no inner ear malformation; } \\
\text { sequenced exon } 2\end{array}$ & $\begin{array}{l}\text { Mixed } \\
N=35\end{array}$ & $\begin{array}{l}2235 \Delta \mathrm{C} / 235 \Delta \mathrm{C} \\
2235 \Delta \mathrm{C} / \mathrm{R} 143 \mathrm{~W} \\
1 \mathrm{G} 45 \mathrm{E} / 299 \Delta \mathrm{AT} \\
1235 \Delta \mathrm{C} /+\end{array}$ & $\begin{array}{l}3235 \Delta \mathrm{C} / \mathrm{Y} 136 \mathrm{X} \\
1 \mathrm{R} 143 \mathrm{~W} / 176 \Delta 16 \\
1 \mathrm{R} 143 \mathrm{~W} / \mathrm{V} 37 \mathrm{I}\end{array}$ & $\begin{array}{l}28.6 \% \\
(14.6-46.3)\end{array}$ \\
\hline & 5 & $\begin{array}{l}\text { Nonsyndromic hearing loss; sequenced } \\
\text { exon } 2\end{array}$ & $\begin{array}{l}\text { Recessive }^{e} \\
N=20\end{array}$ & $\begin{array}{l}3235 \Delta \mathrm{C} / 235 \Delta \mathrm{C} \\
1235 \Delta \mathrm{C} /+\end{array}$ & & $\begin{array}{l}15.0 \% \\
(3.2-37.9 \%)\end{array}$ \\
\hline & 6 & $\begin{array}{l}\text { Prelingual (onset before age } 3 \text { ) hearing } \\
\text { loss, excluding cases of viral } \\
\text { infections, meningitis, or ototoxic } \\
\text { drug use; } 16 \text { females and } 23 \text { males; } \\
\text { sequenced exon } 2\end{array}$ & $\begin{array}{l}\text { Non-familial } \\
N=24\end{array}$ & $\begin{array}{l}1235 \Delta \mathrm{C} / 235 \Delta \mathrm{C} \\
1235 \Delta \mathrm{C} / 176 \Delta 16\end{array}$ & & $\begin{array}{l}8.3 \% \\
(1.0-27.0)\end{array}$ \\
\hline
\end{tabular}


Table 3

(Continued)

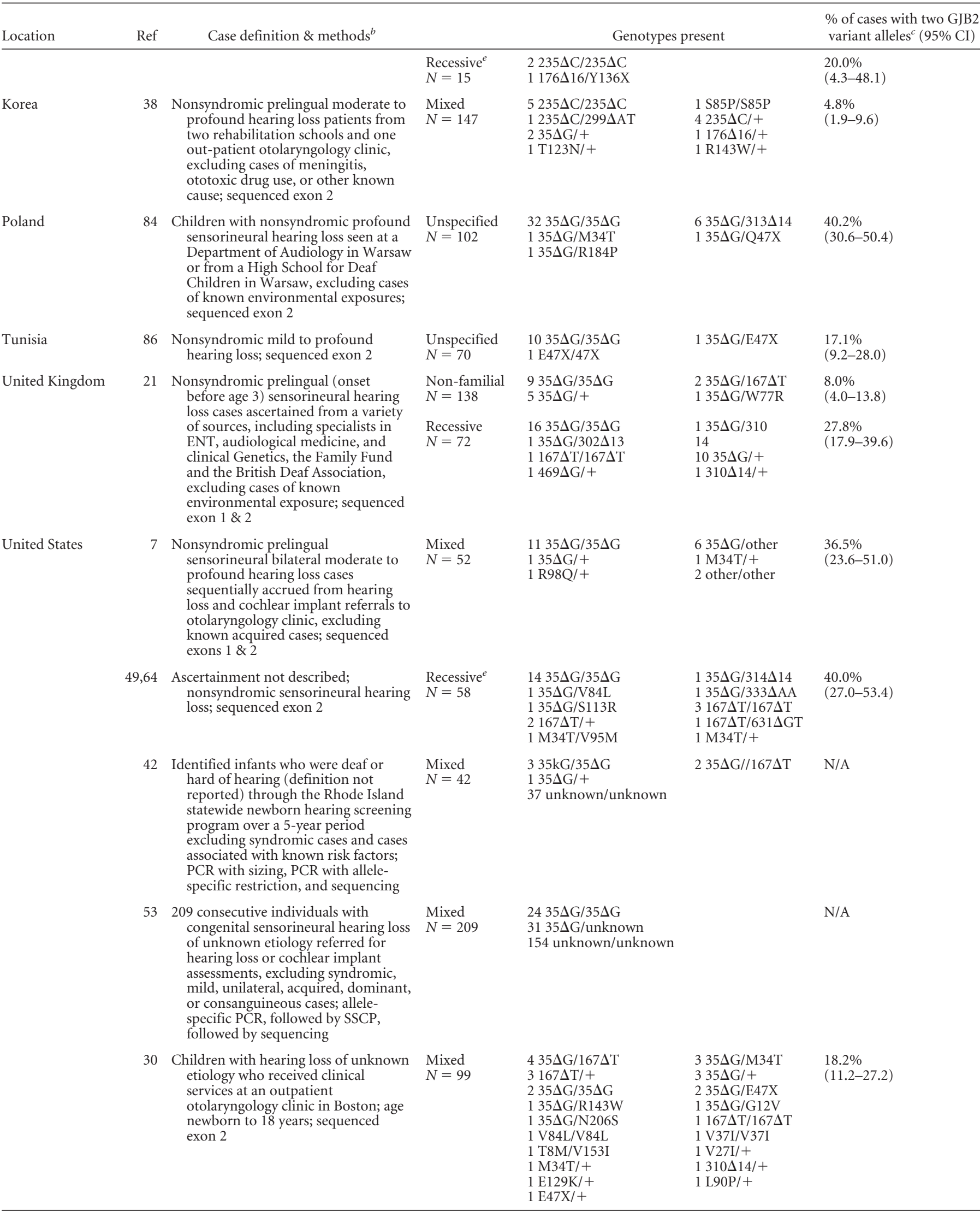


Table 3

(Continued)

\begin{tabular}{|c|c|c|c|c|c|c|}
\hline \multirow[t]{2}{*}{ Location } & \multirow{2}{*}{$\begin{array}{l}\text { Ref } \\
52\end{array}$} & \multicolumn{2}{|l|}{ Case definition \& methods ${ }^{b}$} & \multicolumn{2}{|c|}{ Genotypes present } & \multirow{2}{*}{$\begin{array}{l}\% \text { of cases with two GJB2 } \\
\text { variant alleles }{ }^{c}(95 \% \mathrm{CI}) \\
\text { N/A }\end{array}$} \\
\hline & & $\begin{array}{l}\text { Individuals with hereditary } \\
\text { nonsyndromic hearing loss recruited } \\
\text { from Universities in San Francisco } \\
\text { and Baltimore, excluding cases of } \\
\text { known environmental exposures; } \\
\text { denaturing high-performance liquid } \\
\text { chromatography of exon 2, } \\
\text { confirmed by sequencing }\end{array}$ & $\begin{array}{l}\text { Unspecified } \\
N=154\end{array}$ & $\begin{array}{l}435 \Delta \mathrm{G} / 35 \Delta \mathrm{G} \\
1167 \Delta \mathrm{T} / 167 \Delta \mathrm{T} \\
1504 \text { insAACC/235 } \mathrm{C} \\
24 \text { heterozygotes or } \\
\text { compound } \\
\text { heterozygotes }\end{array}$ & $\begin{array}{l}3 \mathrm{~V} 37 \mathrm{I} / \mathrm{V} 37 \mathrm{I} \\
1299 \Delta \mathrm{AT} / 299 \Delta \mathrm{T}\end{array}$ & \\
\hline Venezuela & 92 & $\begin{array}{l}\text { Children with prelingual sensorineural } \\
\text { hearing loss evaluated for cochlear } \\
\text { implants at two institutions in } \\
\text { Caracas between November } 1998 \\
\text { and May 1999; age less than } 10 \text { years; } \\
\text { SSCP of exon } 2\end{array}$ & $\begin{array}{l}\text { Unspecified } \\
N=42\end{array}$ & $\begin{array}{l}235 \Delta \mathrm{G} / 35 \Delta \mathrm{G} \\
135 \Delta \mathrm{G} / \text { unknown }\end{array}$ & & $\begin{array}{l}4.8 \% \\
(0.6-16.2)\end{array}$ \\
\hline
\end{tabular}

SSCP, single-strand conformation polymorphism; N/A, not applicable (i.e., cannot be calculated with data in this review); CSGE, conformation sensitive electrophoresis.

${ }^{a}$ Nonfamilial cases are cases with no family history of hearing loss. They may represent genetic (e.g., autosomal revessive) or nongenetic cases.

${ }^{b}$ Age and sex unspecified unless noted otherwise.

${ }^{c}$ Excludes carriers of putative polymorphisms: V27I, K41R, S72C, V84A, E114G,I203T. CI, confidence interval.

${ }^{d}$ Overlap of samples?

${ }^{e}$ Recessive cases are individuals with hearing loss who have at least one affected sibling and no affected parents.

\section{Population differences in contribution of GJB2 to hearing loss}

As indicated above, there are population differences in the distribution of the various GJB2 alleles. Despite that different alleles predominate in different populations, there is a relatively high carrier rate of GJB2 alleles in all described populations. Furthermore, the carrier rate seems to be slightly higher in certain geographical areas, such as the Mediterranean region. ${ }^{26,27}$ The cause of this high carrier rate is unknown.

A notable gap in the literature is the lack of assessment of the contribution of GJB2 variants to hearing loss over a wide range of populations, as illustrated by the African American population as described below. Characterization of these populations is important to determine (1) the prevalence of GJB2 variants among individuals with nonsyndromic hearing loss, and (2) the prevalence of the different alleles in the control populations. As demonstrated in Table 3 and discussed above, both of these measures appear to be population-specific.

The proportion of individuals with nonsyndromic hearing loss in African Americans who are carriers of GJB2 variants has not been determined. However, two studies have looked for specific GJB2 variants among African American control groups. The first group consisted of individuals receiving genetic counseling at the University of Michigan for disorders unrelated to hearing loss. This study tested 173 African Americans for the $35 \Delta \mathrm{G}$ variant and 171 African Americans for the $167 \Delta \mathrm{T}$ variant, and found no carriers of either allele. ${ }^{28}$ The other study looked for $35 \Delta G$ variants among 190 African Americans (ascertainment details not reported) and found none. ${ }^{26}$ These two studies indicated that $35 \Delta \mathrm{G}$ is significantly less common among the African American population than it is among the Caucasian population (as described above and in Table 2). The rate of nonsyndromic hearing loss is not lower in African Americans than in Caucasians. ${ }^{29}$ Two possible explanations for these data are (1) the proportion of cases of hearing loss attributed to GJB2 variants is lower in African American than in Caucasian populations, and/or (2) GJB2 alleles other than $35 \Delta G$ play a significant role in the African American population.

In support of the latter model, no individuals with the $35 \Delta \mathrm{G}$ variant were found among 365 students with profound sensorineural hearing loss in Ghana. Likewise, the $167 \Delta \mathrm{T}$ and $235 \Delta \mathrm{C}$ variants were not found in this population. Of the 63 individuals in this study who carried GJB2 variants, 51 (81.0\%) were homozygous for the R143W allele and 8 (12.7\%) were compound heterozygotes for R143W and a second variant allele. ${ }^{25}$ Assessment of GJB2 variants among non-Caucasian hearing loss and control populations are necessary to address these issues so that the clinical validity can be defined in these populations.

\section{Type of hearing loss}

Connexin 26 is expressed in the stria vascularis, spiral ligament, spiral limbus, and between the supporting cells in the cochlea, ${ }^{3}$ and appears to function in the recycling of potassium that is used by the hair cells to generate an action potential in response to sound waves. ${ }^{4}$ Consequently, it has generally been presumed that hearing loss associated with mutations in the GJB2 gene will be sensorineural in nature. The nature of GJB2related hearing loss has not been formally assessed by genetic epidemiologic methods. With one exception, the studies presented in this review either excluded conductive and mixed cases of hearing loss or did not distinguish between the different types of hearing loss. In the study of 99 unrelated children with hearing loss of unknown etiology who were attending an outpatient otolaryngology clinic in Boston, 30 were found to carry one or two GJB2 mutations. Temporal bone abnormalities were identified in four of these individuals $(35 \Delta \mathrm{G} / 167 \Delta \mathrm{T}$, $35 \Delta \mathrm{G} / \mathrm{G} 12 \mathrm{~V}, \mathrm{~L} 90 \mathrm{P} /+$, and $35 \Delta \mathrm{G} /+$ ), and conductive or 
mixed hearing loss was reported for one $(\mathrm{E} 47 \mathrm{X} /+)$ and two (both $35 \Delta \mathrm{G} / \mathrm{M} 34 \mathrm{~T}$ ) cases, respectively. ${ }^{30}$ These associations may be coincidental, but additional studies are needed to describe the type of hearing loss associated with of GJB2 variants.

\section{Age of onset of hearing loss}

GJB2 variants are generally described as causing prelingual hearing loss. However, in most published studies, it is not possible to distinguish between congenital (present at birth) and noncongenital prelingual hearing loss. Only one published study has examined the contribution of GJB2 variants to congenital hearing loss. The prevalence of the $35 \Delta \mathrm{G}$ genotypes in a Rhode Island newborn population with hearing loss did not differ from other American populations with hearing loss who were ascertained in childhood and who were of similar race/ ethnicity (Table 3). More studies of this type, as well as studies including documented noncongenital prelingual hearing loss, are needed to assess the relationship between GJB2 variants and congenital hearing loss. In this regard, the reports of newborns who passed the newborn hearing screen but in whom GJB2-related hearing loss was diagnosed later in infancy are notable. ${ }^{31,32}$ Whether these cases represented false-negative results of the newborn hearing screening programs or indicated a late-onset and/or progressive nature of some GJB2-related cases of hearing loss is not clear. Likewise, Orzan et al. reported three Italian children with biallelic GJB2 genotypes who had a sudden onset of hearing loss between 18 and 24 months of age, although it is not clear whether prior hearing status was formally documented or based on parental report. ${ }^{23}$

Recent research has not focused on rigorous analysis of the possible contribution of $G J B 2$ to postlingual hearing loss. Four published studies have included individuals with postlingual hearing loss. The first consisted of genetic analysis of GJB2 among individuals recruited from consecutive patients at the genetic counseling service for deaf individuals at two hospitals in Paris. Of the participants, 43 of the 88 individuals with prelingual sensorineural hearing loss carried variations in the GJB2 gene, but no changes were found among the 16 individuals with postlingual (before age 20) sensorineural hearing loss. ${ }^{20}$ Likewise, a study in Israel ascertained individuals with nonsyndromic hearing loss (ascertainment details not reported) and tested them for GJB2 variants. Of the 66 individuals with prelingual hearing loss, 25 were homozygous or compound heterozygous for GJB2 variants, and 4 were heterozygous. No GJB2 variants were found among the 11 cases of postlingual (definition not provided) hearing loss. ${ }^{24}$ In Japan, 5 of 39 individuals with prelingual hearing loss were homozygous or heterozygous GJB2 variant carriers, but no changes were found among the 39 individuals with postlingual (onset between 3 and 30 years) sensorineural hearing loss (ascertainment details not reported). ${ }^{6}$

The fourth study, taking place in Austria, found four carriers of GJB2 variants among 16 individuals with postlingual (undefined) hearing loss. ${ }^{22}$ The genotypes were L90P/I20T (onset in first decade), $\mathrm{L} 90 \mathrm{P} / 35 \Delta \mathrm{G}$ (onset in first decade), $35 \Delta \mathrm{G} /+$ (onset in first decade), and G160S/+ (onset in fourth decade). The
L90P allele is of interest in this population because it is seen in 2 of 16 postlingual (undefined) cases, and 3 of 53 prelingual cases of hearing loss. Thus this allele may be a significant contributor to postlingual hearing loss. The failure to detect GJB2 variants in the other three studies may be due to a higher prevalence of the L90P allele in the Austrian population, as this allele was detected only rarely in individuals with hearing loss in France ( 2 of 135$)^{20,35}$ but not at all in Israel $(0 \text { of } 102)^{24,33}$ or Japan (0 of 94).5,6,34

Two dominant alleles have been specifically implicated in noncongenital hearing loss. The $\mathrm{C} 202 \mathrm{~F}$ allele was observed to cosegregate with postlingual (age of onset at 10 to 20 years) and progressive hearing loss in a 5-generation French family. ${ }^{36}$ Likewise, the W44C allele cosegregated with progressive hearing loss in an American family of mixed Northern European descent, with age of onset ranging from infancy to age 18 years. ${ }^{37}$ These alleles were not detected in studies that provided sequence data on controls, including 100 Korean newborns, ${ }^{38}$ 209 Japanese individuals, ${ }^{5,6,34}$ and 204 French individuals. ${ }^{36,35}$

These studies suggest that hearing loss associated with the more common GJB2 sequence variations is likely to be prelingual. However, additional population-based studies involving individuals with congenital, noncongenital prelingual, postlingual, and late-onset hearing loss will be needed to fully assess the relationship between GJB2 variants and age of onset, particularly in reference to the less common alleles.

\section{Severity of hearing loss}

Hearing loss associated with GJB2 variations generally fall into the moderate to profound range. Three European studies have looked at the severity of hearing loss among children with and without $G J B 2$ sequence changes. In one of these studies, in France (ascertainment described above), GJB2 homozygotes or compound heterozygotes accounted for 31 (55\%) of 56 profound ( $\geq 90 \mathrm{~dB})$ cases, $14(48 \%)$ of 29 severe $(70-89 \mathrm{~dB})$ cases, $8(42 \%)$ of 19 moderate $(40-69 \mathrm{~dB})$ cases, and $1(14 \%)$ of 7 mild $(20-39 \mathrm{~dB})$ cases..$^{20}$ Of the 47 individuals who carried the $35 \Delta \mathrm{G} / 35 \Delta \mathrm{G}$ genotype, the hearing loss was profound in 29 $(62 \%)$, severe in $10(21 \%)$, moderate in $7(15 \%)$, and mild in 1 (2\%). ${ }^{39}$ The profile for individuals with one $35 \Delta \mathrm{G}$ and one other allele was two profound (22\%), three moderate (33\%), three severe (33\%), and one mild (11\%) Although the latter group is small in size, the results are suggestive of variability in degree of hearing loss between alleles.

In 1999, a United Kingdom (U.K.) group ascertained a group of 284 individuals with nonsyndromic prelingual sensorineural hearing loss from several sources, including otolaryngologists, audiologists, clinical geneticists, and the British Deaf Associations. ${ }^{21}$ They found biallelic GJB2 carriers among 0 $(0 \%)$ of 19 mild $(20-39 \mathrm{~dB})$ cases, $9(10 \%)$ of 92 moderate $(40-69 \mathrm{~dB})$ cases, $11(17 \%)$ of 64 severe $(70-94 \mathrm{~dB})$ cases, and $30(30 \%)$ of 100 profound ( $\geq 95 \mathrm{~dB}$ ) cases. The $35 \Delta \mathrm{G} / 35 \Delta \mathrm{G}$ genotype was present in 6 individuals with moderate, 10 with severe, and 24 with profound hearing loss. Only two $167 \Delta \mathrm{T} /$ $167 \Delta \mathrm{T}$ individuals were found in this study, and both had 
moderate hearing loss. Two $35 \Delta \mathrm{G} / 167 \Delta \mathrm{T}$ individuals were found: one with moderate and one with severe hearing loss.

Also in 1999, 94 individuals with nonsyndromic prelingual hearing loss were recruited from Italian audiology and phoniatrics services. Of these individuals with profound hearing loss ( $\geq 95 \mathrm{~dB}$ ), $63 \%$ carried GJB2 variant alleles as did $43 \%$ of individuals with severe (70-94 dB) and 33\% of those with moderate ( $40-69 \mathrm{~dB})$ hearing loss. Of the individuals with GJB2 variant genotypes, 27 were homozygous for $35 \Delta G$ and 13 were compound heterozygotes. As in the French study discussed above, the $35 \Delta \mathrm{G}$ homozygotes fell into the moderate or profound range, whereas the compound heterozygotes were dispersed among the three categories (moderate, severe, and profound), suggesting allelic difference in expressivity. ${ }^{23}$

Seven additional studies presented data regarding the severity of hearing loss among individuals with GJB2 variants. Because the number of alleles and, therefore, the number of possible genotypes was large, the absolute numbers of cases for each genotype in the studies combined were small. Therefore, the data presented here focus on the $35 \Delta \mathrm{G} / 35 \Delta \mathrm{G}$ and $167 \Delta \mathrm{T} /$ $167 \Delta$ T genotypes. Two Israeli studies, ${ }^{24,33}$ two Australian studies, ${ }^{40,41}$ one Austrian study, ${ }^{22}$ and two American studies ${ }^{30,42}$ described the level of hearing loss among individuals with GJB2 mutations. In the seven studies combined, information was presented on 50 people with the $35 \Delta \mathrm{G} / 35 \Delta \mathrm{G}$ genotype: 8 moderate (16\%), 12 severe (24\%), and 30 profound $(60 \%)$. Likewise, of the 30 people with the $167 \Delta \mathrm{T} / 167 \Delta \mathrm{T}$ genotype, one had mild hearing loss (3.3\%), 5 moderate (16.7\%), 8 severe $(26.7 \%)$, and 16 profound $(53.3 \%)$. These data are consistent with the above reports, and indicate that the hearing loss associated with the $35 \Delta \mathrm{G} / 35 \Delta \mathrm{G}$ and $167 \Delta \mathrm{T} / 167 \Delta \mathrm{T}$ genotypes is generally in the moderate to profound range, with profound hearing loss being the most common manifestation.

Although these data suggest that GJB2 variants tend to be associated with moderate to profound hearing loss, the numbers were small, $\mathrm{dB}$ ranges of degrees of hearing loss varied among the studies, and the specific relationship between various GJB2 alleles and severity of hearing loss were not addressed. In addition, the nonpopulation-based approach may have resulted in underascertainment of mild hearing loss. However, the low prevalence of GJB2 biallelic genotypes among the individuals with mild hearing loss in the British ${ }^{21}$ and French ${ }^{20}$ studies described above suggested that GJB2-associated hearing loss, particularly with the $35 \Delta \mathrm{G}$ and $167 \Delta \mathrm{T}$ alleles, tends to be moderate to profound. Likewise, GJB2 biallelic individuals have not been described in the general hearing population. On the other hand, the Australian group described three individuals with mild hearing loss $(25-40 \mathrm{~dB})$ with less common GJB2 genotypes: M34T/R184W, 35 $\Delta$ G/M34T, and $35 \Delta \mathrm{G} / \mathrm{V} 37 \mathrm{I} .{ }^{40}$ Likewise, the Austrian study reported three individuals with mild hearing loss: $\mathrm{L} 90 \mathrm{P} / 314 \Delta 14, \mathrm{Y} 155 \mathrm{X} /+$, and G160S/+. ${ }^{22}$ It is possible that carriers of these alleles do not always have hearing loss, but because these alleles are less common than the $35 \Delta \mathrm{G}$ and $167 \Delta \mathrm{T}$ alleles, larger populationbased studies are needed to address this issue.

\section{Laterality of hearing loss}

The inter-ear difference in severity of hearing loss was described for 54 French children with biallelic GJB2 genotypes. In $48(89 \%)$ of the children, the severity did not differ between the ears. In the other six (11\%), the ears differed by one degree of severity ( $\mathrm{dB}$ ranges described above). ${ }^{20}$ These children were ascertained through genetic counseling services for deaf individuals at two hospitals in Paris. Therefore, individuals with unilateral hearing loss may have been underascertained. The study included two children with two degrees, and one child with three degrees of difference in severity, none of whom carried GJB2 variants. However, the number of children in these groups was clearly small.

In an analysis of children with nonsyndromic prelingual hearing loss ascertained through Italian audiology services, more than $90 \%$ of the 46 children with GJB2 variants demonstrated a symmetrical hearing loss (inter-ear difference of $<15$ $\mathrm{dB}$ at two frequencies or $10 \mathrm{~dB}$ at four frequencies). In this study, GJB2 variants were detected in 43 of 75 (66\%) individuals with symmetrical hearing loss but in only four of the 19 (21\%) of those with asymmetrical hearing loss. ${ }^{23}$

In the U.K. study, ${ }^{21}$ individuals were ascertained through a variety of sources, including otolaryngologists, clinical geneticists, and Deaf associations. The data were presented as average $\mathrm{dB}$ difference in hearing loss between the ears: $6.33(N=45$, $\mathrm{SD}=8.09)$ for $G J B 2$ homozygotes and compound heterozygotes, $6.66(N=26, \mathrm{SD}=8.05)$ for heterozygotes, and $7.86(N$ $=175, \mathrm{SD}=11.19)$ for individuals without GJB2 variations. There was no significant difference between any of these groups.

In a group of consecutive individuals with sensorineural hearing loss seen at a center for Hearing, Speech, and Voice Disorders in Austria, 24 individuals with GJB2 variant genotypes were identified. Of these, five displayed asymmetry of hearing loss: three by two degrees and two by one degree of severity. ${ }^{22}$

In all of these studies, the ascertainment of individuals with unilateral hearing loss is unclear. Therefore, although the described GJB2 variants tend to be associated with bilateral hearing loss, population-based data on all individuals with any type of hearing loss are needed to clarify the issue.

\section{Progression of hearing loss}

Longitudinal data are lacking on individuals with GJB2-related hearing loss. Follow-up on individuals with GJB2-associated hearing loss over 1 to 20 years indicated no changes in the threshold of hearing loss. ${ }^{43}$ However, details about the number of such cases and the timing of repeated testing were not published.

The French group studied children ascertained through genetic counseling services for the deaf in Paris, and described 16 children with biallelic GJB2 genotypes for whom test results were available over a 10-year span. In 11 children, no change $(\leq 5 \mathrm{~dB})$ in the threshold was noted. Three children (one with severe and two with profound hearing loss) showed slight pro- 
gression (5-10 dB). Two children (one with moderate to severe, and one with profound hearing loss) had a progression $>10 \mathrm{~dB} \cdot{ }^{20}$

Likewise, a retrospective analysis of audiograms (over 2 to 15 years) in Italian children ascertained through audiology services detected a progression of hearing loss in only 1 child of 47 who had a GJB2 variant genotype. Progression was defined as a $>15 \mathrm{~dB}$ change in two or more frequencies, or a $>10 \mathrm{~dB}$ change over an average of four frequencies. ${ }^{23}$

Among 24 Austrian individuals with GJB2-related sensorineural hearing loss, 3 were described as progressive in nature, although the definition was not provided.22 Thus the limited data suggest the GJB2-related hearing loss is primarily nonprogressive in nature.

\section{High-frequency hearing loss}

Audiograms of individuals with biallelic GJB2 genotypes tend to be flat or slightly descending, indicating equal loss across all frequencies. ${ }^{20-24,38,40,41,43-47}$ Two individuals with the $35 \Delta \mathrm{G} / \mathrm{L} 90 \mathrm{P}$ genotype have been described with high-frequency $(2,000-8,000 \mathrm{~Hz})$ hearing loss. ${ }^{40}$ This finding suggested that certain alleles, other than the more common and well-studied $35 \Delta \mathrm{G}$ and $167 \Delta \mathrm{T}$, may be associated with highfrequency hearing loss. Most individuals with GJB2 variants have been ascertained through services for individuals with hearing loss, and many of the studies did not assess the highfrequency $(2,000-8,000 \mathrm{~Hz})$ range. Thus the contribution of GJB2 variants to high-frequency-only hearing loss has not been well-studied.

\section{M34T allele and hearing loss}

In 1997, the M34T variant was reported to cosegregate with three generations of hearing loss in one family in an apparently dominant manner, implicating GJB2 in nonsyndromic hearing loss. ${ }^{3}$ Several years later, a second variant was characterized in this family, found in trans with the M34T allele in the individuals with hearing loss. This finding suggested that the M34T allele is recessive. ${ }^{48}$ On the other hand, the M34T allele failed to cosegregate with hearing loss in several families, raising the possibility that M34T is a benign polymorphism. ${ }^{36,49-51}$

The prevalence of M34T heterozygote carriers and compound heterozygotes among individuals with hearing loss and control groups is summarized in Table 4. The M34T allele is present in approximately $2 \%$ to $3 \%$ of the general Caucasian population, but has not been reported in Japan or Korea. Data on other populations are limited.

A similar prevalence of the M34T alleles was seen among Caucasian individuals with hearing loss, supporting the model that the M34T allele is a benign polymorphism. However, the $\mathrm{M} 34 \mathrm{~T}$ allele was sometimes found as a compound heterozygote among individuals with hearing loss, 7,30,34,39,46,49-52 and more rarely, in the homozygous form in individuals with hearing loss. ${ }^{39,51,53}$ No changes have been reported in the other allele among the M34T carriers in the control groups. Thus, the evidence appears to support the hypothesis that M34T is a recessive allele, although the lack of compound heterozygotes in the control groups may be due to their smaller sample sizes. This example demonstrates the importance of looking at data on genotypes rather than allele frequency. In addition, a recent report of linkage between M34T and an upstream 10-base pair deletion raised the possibility that the association between M34T and hearing loss may be due to linkage disequilibrium. ${ }^{51}$ Additional studies are needed to clarify the involvement of the M34T allele in hearing loss.

\section{GJB2 variants and Vohwinkel syndrome}

The vast majority of GJB2 variants are associated with nonsyndromic hearing loss (see Table 1). Ironically, the original report of a GJB2 allele associated with hearing loss occurred in a family that also displayed palmoplantar keratoderma (PPK). PPK is a form of hyperkeratosis in which the overgrowth is limited to the palms of the hands and the soles of the feet. The M34T allele cosegregated with hearing loss but not PPK in this family. ${ }^{3}$ Subsequently, it was shown that the PPK in this family was due to a $\mathrm{D} 66 \mathrm{H}$ variant in the GJB2 gene, a variant not seen in the 122 unrelated controls. ${ }^{48}$ The combination of dominant sensorineural hearing loss and hyperkeratosis is also known as Vohwinkel syndrome (OMIM: 124500), and hearing loss with PPK appears to be a mild variant. The D66H allele has been implicated in this syndrome in three additional families. ${ }^{54} \mathrm{Nei}-$ ther group detected the $\mathrm{D} 66 \mathrm{H}$ allele among the control groups of 122 and 145 unrelated individuals.

Likewise, the G59A variant cosegregated with Vohwinkel syndrome in a three-generation family. The G59A allele was not detected among 50 hearing controls or among 55 individuals with nonsyndromic hearing loss. .55

The R75W variant was described in an Egyptian family with autosomal PPK and congenital deafness. It was also detected in one individual in the control group of 77 Egyptian individuals attending a clinic for reasons unrelated to skin disorders; however, the hearing status of this control individual is unknown. $\mathrm{R} 75 \mathrm{~W}$ was not found in the 17 Caucasian controls. ${ }^{56}$

Several other studies included sequence information about control individuals, and none of them detected any carriers of D66H, G59A, or R75W. The studies included 100 Korean newborns, ${ }^{38} 209$ Japanese individuals, ${ }^{5,6,34}$ and 119 French individuals $^{35}$ (ascertainment details provided in Table 3). The numbers of controls examined in these studies were small and did not necessarily come from the same population as the cases; therefore, we cannot rule out the possibility that these alleles may present in the general population at a low frequency due to incomplete penetrance.

\section{INTERACTIONS}

Many study groups reported variations in the degree of hearing loss in individuals with the same genotype (see the Severity of Hearing Loss section above), even within sibships. ${ }^{20,22,24,33,41,43}$ For example, one Israeli family consisted of five siblings with the $167 \Delta \mathrm{T} / 167 \Delta \mathrm{T}$ genotype; three had profound $(\geq 90 \mathrm{~dB})$, one had severe $(70-89 \mathrm{~dB})$, and one had mild (20-39 dB) hearing loss. ${ }^{33}$ Likewise, in the French report of 16 
Kenneson et al.

Table 4

Prevalence of M34T heterozygote carriers and compound heterozygotes in cases and controls in various geographical areas (raw numbers, frequencies, and 95\% confidence intervals)

\begin{tabular}{|c|c|c|c|c|c|}
\hline \multirow[b]{2}{*}{ Location } & \multirow[b]{2}{*}{ Reference } & \multicolumn{2}{|c|}{ General population } & \multicolumn{2}{|c|}{ People with hearing loss } \\
\hline & & M34T/+ & M34T/other & $\mathrm{M} 34 \mathrm{~T} /+$ & M34T/other \\
\hline \multirow[t]{9}{*}{ France } & 20 & & & $0 / 88$ & $0 / 88$ \\
\hline & & & & $0 \%$ & $0 \%$ \\
\hline & & & & $(0-4.1)$ & $(0-4.1)$ \\
\hline & 7 & $3 / 128$ & $0 / 128$ & & \\
\hline & & $2.3 \%$ & $0 \%$ & & \\
\hline & & $(0.5-6.7)$ & $(0-2.8)$ & & \\
\hline & 39 & $1 / 116$ & $0 / 116$ & $3 / 96$ & $1 / 96$ \\
\hline & & $0.9 \%$ & $0 \%$ & $3.1 \%$ & $1.0 \%$ \\
\hline & & $(0.0-4.7)$ & $(0-3.1)$ & $(0.6-8.9)$ & $(0.0-5.7)$ \\
\hline \multirow[t]{6}{*}{ Japan } & 34 & $0 / 96$ & $0 / 96$ & $0 / 35$ & $0 / 35$ \\
\hline & & $0 \%$ & $0 \%$ & $0 \%$ & $0 \%$ \\
\hline & & $(0-3.8)$ & $(0-3.8)$ & $(0-10.0)$ & $(0-10.0)$ \\
\hline & 6 & $0 / 63$ & $0 / 63$ & $0 / 39$ & $0 / 39$ \\
\hline & & $0 \%$ & $0 \%$ & $0 \%$ & $0 \%$ \\
\hline & & $(0-5.7)$ & $(0-5.7)$ & $(0-9.0)$ & $(0-9.0)$ \\
\hline \multirow[t]{3}{*}{ Korea } & 38 & $0 / 100$ & $0 / 100$ & $0 / 147$ & $0 / 147$ \\
\hline & & $0 \%$ & $0 \%$ & $0 \%$ & $0 \%$ \\
\hline & & $(0-3.6)$ & $(0-3.6)$ & $(0-2.5)$ & $(0-2.5)$ \\
\hline \multirow{9}{*}{$\begin{array}{l}\text { United Kingdom \& } \\
\text { Ireland }\end{array}$} & 51 & $25 / 630$ & $0 / 630$ & $7 / 173$ & $3 / 173$ \\
\hline & & $4.0 \%$ & $0 \%$ & $4.0 \%$ & $1.7 \%$ \\
\hline & & $(2.6-5.8)$ & $(0-0.6)$ & $(1.6-8.2)$ & $(0.4-5.0)$ \\
\hline & 48 & $0 / 40$ & $0 / 40$ & & \\
\hline & & $0 \%$ & $0 \%$ & & \\
\hline & & $(0-8.8)$ & $(0-8.8)$ & & \\
\hline & 21 & & & $0 / 210$ & $0 / 210$ \\
\hline & & & & $0 \%$ & $0 \%$ \\
\hline & & & & $(0-1.7)$ & $(0-1.7)$ \\
\hline \multirow[t]{19}{*}{ United States } & 7 & & & $1 / 52$ & $1 / 52$ \\
\hline & & & & $1.9 \%$ & $1.9 \%$ \\
\hline & & & & $(0.1-10.3)$ & $(0.1-10.3)$ \\
\hline & 49 & $3 / 96$ & $0 / 96$ & & \\
\hline & & $3.1 \%$ & $0 \%$ & & \\
\hline & & $(0.6-8.9)$ & $(0.3-8)$ & & \\
\hline & 30 & & & $1 / 30$ & $3 / 30$ \\
\hline & & & & $3.3 \%$ & $10.0 \%$ \\
\hline & & & & $(0.1-17.2)$ & $(2.1-26.5)$ \\
\hline & 52 & & & & \\
\hline & & & & & \\
\hline & & & & & \\
\hline & & & & & \\
\hline & 53 & & & $0 / 209$ & $2 / 209$ \\
\hline & & & & $0 \%$ & $1.0 \%$ \\
\hline & & & & $(0-1.8)$ & $(0.1-3.4)$ \\
\hline & 74 & $1 / 100$ & $0 / 100$ & & \\
\hline & & $1.0 \%$ & $0 \%$ & & \\
\hline & & $(0.0-5.4)$ & $(0-3.6)$ & & \\
\hline
\end{tabular}

${ }^{a}$ Publication did not distinguish between heterozygotes and compound heterozygotes. 
children with biallelic GJB2 genotypes, the degree of hearing loss differed between the siblings in $50 \%$ of the families. ${ }^{20}$ This finding suggests that other factors, genetic and/or environmental, may be modifying the phenotypic outcome.

Individuals who are carriers of a single variation in GJB2 display evidence of reduced hair cell function ${ }^{57}$; therefore, it is possible that these individuals are more likely to develop hearing loss in the presence of additional genetic or environmental factors than are noncarriers. This possibility is supported by recent reports of mutations in other genes found at increased incidence among GJB2 carriers with hearing loss: GJB6 (connexin 30$)^{58}$ and the mitochondrial $12 \mathrm{~S}$ rRNA. ${ }^{59}$ Additional studies of this nature are expected to continue to characterize the gene-gene interactions involved in the etiology of GJB2associated hearing loss.

Many of the studies in this review excluded cases of suspected environmental causes from the genetic analysis. These factors included infections (e.g., meningitis, rubella), low birth weight, ventilator use, ototoxic medications (e.g., aminoglycosides), and hyperbilirubinemia. However, two individuals with hearing loss attributed to rubella infection were later found to be homozygous for the $167 \Delta \mathrm{T}$ variant. ${ }^{32}$ Thus, the presence of known environmental factors does not necessarily preclude genetic analysis. Indeed, the proportion of GJB2 cases that have been attributed to other causes has not been elucidated; therefore, the possibility of gene-environment interactions has not been examined.

Likewise, published studies have generally excluded cases of syndromic hearing loss from GJB2 analysis. Thus the possibility that GJB2 variants may be involved in the penetrance and expressivity of hearing loss due to syndromic causes has not been examined.

\section{LABORATORY TESTS}

Many DNA-based methods are available for detecting the various alleles reported for the GJB2 gene. Assays have been developed to rapidly test for specific common variants, including allele-specific polymerase chain reaction (PCR), PCR followed by restriction enzyme digestion, and PCR with allelespecific hybridization. These technologies, once analytically validated in the performing laboratory, are both highly sensitive and specific. However, they will only detect the allele for which they were designed. As some common alleles account for the majority of variants in some populations (e.g., $35 \Delta \mathrm{G}$ in Greece), these methods offer rapid and economical approaches. They also provide simple and reliable methods for carrier testing in families with known alleles.

Scanning methodologies are often used for allele detection, including denaturing gradient gel electrophoresis, singlestrand conformation polymorphism detection, heteroduplex analysis, and denaturing high-performance liquid chromatography. Although scanning technologies have the advantage of screening for many variants at once, they tend to be less reliable than the allele-specific PCR-based techniques in that they are more sensitive to laboratory conditions. They also will miss some alleles, the specific alleles being detected dependent on the method and conditions.

Sequencing of PCR products of the GJB2 gene is a common approach that has the advantage of detecting most alleles, including novel changes. Of the 94 known variants described in Table 1 , all but 2 are in exon 2. Both exons 1 and 2 are small and amenable to PCR amplification. Sequencing of exon 1 will pick up these remaining three alleles. As described in the Contribution of GJB2 to Hearing Loss section, only a few published studies have used the method of sequencing both exons 1 and 2. Therefore, information is lacking to accurately determine the relative clinical validity of these two methods.

Laboratories offering clinical testing for GJB2 vary in their methodologies of choice (Kenneson et al., unpublished). Clinical validity thus varies accordingly.

\section{POPULATION TESTING}

Consistent with the recommendations of the Joint Committee on Infant Hearing, ${ }^{60}$ a growing number of states are screening newborns for audiologic function so that infants with hearing loss are identified and referred for intervention services very early in life. Some newborn hearing screening programs may in the near future refer individuals for genetic testing for GJB2 variants as part of follow-up services. The role that GJB2 testing will play in conjunction with universal newborn hearing screening programs has not yet been defined. Population-based studies are needed to determine the contribution of GJB2 variants to congenital hearing loss, as well as the association between GJB2 variants and progressive hearing loss.

A continuing challenge for laboratories has been the interpretation of novel sequence variants that may have clinical relevance. In recognition of the need, the American College Medical Genetics (ACMG) has published recommendations for interpreting sequence variants of questionable clinical relevance. ${ }^{61}$ The report cautions laboratorians to develop any interpretation made based on what is known not only about the sequence variant but also the individual's chance of having the condition, family history, other test results, and the sensitivity and specificity of the test being performed. As GJB2 testing is used more often in the evaluation of children with hearing loss, interpretation of uncommon and novel mutations will be necessary.

Genetic tests are often offered for clinical use before the clinical validity and utility are fully understood. ${ }^{62,63}$ Because this is the case for GJB2 testing, research participants need to understand the distinction between genetic research, testing, and screening. The identification of GJB2 variants in infants with hearing loss may prove to have many clinical purposes, including (1) ruling-out risk of syndromic complications, (2) predicting moderate to profound hearing loss requiring aggressive language intervention, (3) indicating sensorineural hearing loss for which cochlear implants may be an intervention option for consideration, and (4) allowing genetic counseling regarding recurrence rates. ${ }^{42,64,65}$ The current literature 
is not sufficient for a careful review of all of these potential uses of GJB2 testing. Consequently, the child's course of intervention may not be significantly altered by the knowledge of GJB2 genotype at the present time. Although the genetic information may be useful to the family, genetic testing of minors is generally not accepted in the absence of direct intervention benefits for the child. ${ }^{66}$ However, as more information is collected about GJB2-related hearing loss, and the above-mentioned potential uses are evaluated, GJB2 testing may find a place in medical services that goes beyond reproductive counseling issues.

Genetic testing related to hearing loss is particularly ridden with complex ethical issues. For example, although the ACMG recommends providing genetic services to individuals with hearing loss "to establish the etiology whenever possible," 67 individuals with hearing loss often argue that genetic testing will devalue individuals with hearing loss. ${ }^{68}$ Furthermore, people with hearing loss often have different attitudes and beliefs about genetic testing for hearing loss which in most cases is reflective of different perspectives. One study surveyed parents with normal hearing who have one or more deaf children and demonstrated an overwhelmingly positive attitude toward genetic testing for hearing loss (96\%). ${ }^{69}$ On the contrary, a survey administered to a group of delegates attending a conference on the "Deaf Nation" reported that $55 \%$ thought that genetic testing would do more harm than good and $46 \%$ responded that its potential use devalued people with hearing loss. ${ }^{68}$ The issues raised by the Deaf community provide a unique opportunity by challenging scientists and society to find culturally sensitive methods for genetic research and testing that are acceptable to all cultural groups.

\section{Acknowledgment}

This project was supported in part under a cooperative agreement from the Centers for Disease Control and Prevention through the Association of Teachers of Preventive Medicine.

\section{References}

1. National Association of the Deaf. What is wrong with the use of these terms: "deafmute," "deaf and dumb," or "hearing-impaired?" www.nad.org/infocenter/infotogo/dcc/terms.html

2. Israel J. Psychological aspects of deafness: perspectives. In: Israel J, editor. An introduction to deafness: a manual for genetic counselors. Washington DC: Gallaudet University, 1995:14-179.

3. Kelsell DP, Dunlop J, Stevens HP, Lench NJ, Liang JN, Parry G, Mueller RF, Leigh IM. Connexin 26 mutations in hereditary non-syndromic sensorineural deafness. Nature 1997;387:80-83.

4. Kikuchi T, Adams JC, Paul DL, Kimura RS. Gap junction systems in the rat vestibular labyrinth: immunohistochemical and ultrastructural analysis. Acta Otolaryngol 1994;114:520-528.

5. Fuse Y, Doi K, Hasegawa T, Sugii A, Hibino H, Kubo T. Three novel connexin26 gene mutations in autosomal recessive non-syndromic deafness. Neuroreport 1999; 10:1853-1857.

6. Kudo T, Ikeda K, Kure S, Matsubara Y, Oshima T, Watanabe KI, Kawase T, Narisawa K, Takasaka T. Novel mutations in the connexin 26 gene (GJB2) responsible for childhood deafness in the Japanese population. Am J Med Genet 2000;90:141-145.

7. Green GE, Scott DA, McDonald JM, Woodworth GG, Sheffield VC, Smith RJ. Carrier rates in the midwestern United States for GJB2 mutations causing inherited deafness. JAMA 1999;281:2211-2216.
8. Antoniadi T, Pampanos A, Petersen MB. Prenatal diagnosis of prelingual deafness: carrier testing and prenatal diagnosis of the common GJB2 35delG mutation. Prenat Diagn 2001;21:10-13.

9. Hudspeth AJ. The cellular basis of hearing: the biophysics of hair cells. Science 1985;230:745-752.

10. Davidson J, Hyde ML, Alberti PW. Epidemiologic patterns in childhood hearing loss: a review. Int J Pediatr Otorhinolaryngol 1989;17:239-266.

11. Marazita ML, Ploughman LM, Rawlings B, Remington E, Arnos KS, Nance WE Genetic epidemiological studies of early-onset deafness in the U.S. school-age population. Am J Med Genet 1993;46:486-491.

12. Morton NE. Genetic epidemiology of hearing impairment. Ann N Y Acad Sci 1991; 630:16-31.

13. Van Camp G, Willems PJ, Smith RJ. Nonsyndromic hearing impairment: unparalleled heterogeneity. Am J Hum Genet 1997;60:758-764.

14. Hereditary Hearing Loss Homepage, dnalab-www.uia.ac.be/dnalab/hhh/index.html

15. Hutchin TP, Cortopassi GA. Mitochondrial defects and hearing loss. Cell Mol Life Sci 2000;57:1927-1937.

16. Guilford P, Ben Arab S, Blanchard S, Levilliers J, Weissenbach J, Belkahia A, Petit C. A non-syndrome form of neurosensory, recessive deafness maps to the pericentromeric region of chromosome 13q. Nat Genet 1994;6:24-28.

17. Chaib H, Lina-Granade G, Guilford P, Plauchu H, Levilliers J, Morgon A, Petit C. A gene responsible for a dominant form of neurosensory non-syndromic deafness maps to the NSRD1 recessive deafness gene interval. Hum Mol Genet 1994;3:22192222 .

18. Denoyelle F, Lina-Granade G, Plauchu H, Bruzzone R, Chaib H, Levi-Acobas F, Weil D, Petit C. Connexin 26 gene linked to a dominant deafness. Nature 1998;393: 319-320.

19. Nance WE, Liu XZ, Pandya A. Relation between choice of partner and high frequency of connexin-26 deafness. Lancet 2000;356:500-501.

20. Denoyelle F, Marlin S, Weil D, Moatti L, Chauvin P, Garabedian EN, Petit C. Clinical features of the prevalent form of childhood deafness, DFNB1, due to a connexin-26 gene defect: implications for genetic counselling. Lancet 1999;353:1298 1303.

21. Mueller RF, Nehammer A, Middleton A, Houseman M, Taylor GR, Bitner-Glindzciz M, Van Camp G, Parker M, Young ID, Davis A, Newton VE, Lench NJ. Congenital non-syndromal sensorineural hearing impairment due to connexin 26 gene mutations-molecular and audiological findings. Int J Pediatr Otorhinolaryngol 1999; 50:3-13.

22. Loffler J, Nekahm D, Hirst-Stadlmann A, Gunther B, Menzel HJ, Utermann G, Janecke AR. Sensorineural hearing loss and the incidence of Cx26 mutations in Austria. Eur J Hum Genet 2001;9:226-230.

23. Orzan E, Polli R, Martella M, Vinanzi C, Leonardi M, Murgia A. Molecular genetics applied to clinical practice: the Cx26 hearing impairment. Br J Audiol 1999;33:291295.

24. Sobe T, Vreugde S, Shahin H, Berlin M, Davis N, Kanaan M, Yaron Y, Orr-Urtreger A, Frydman M, Shohat M, Avraham KB. The prevalence and expression of inherited connexin 26 mutations associated with nonsyndromic hearing loss in the Israeli population. Hum Genet 2000;106:50-57.

25. Hamelmann C, Amedofu GK, Albrecht K, Muntau B, Gelhaus A, Brobby GW Horstmann RD. Pattern of connexin 26 (GJB2) mutations causing sensorineural hearing impairment in Ghana. Hum Mutat 2001;18:84-85.

26. Gasparini P, Rabionet R, Barbujani G, Melchionda S, Petersen M, Brondum-Nielsen K, Metspalu A, Oitmaa E, Pisano M, Fortina P, Zelante L, Estivill X. High carrier frequency of the 35delG deafness mutation in European populations. Genetic Analysis Consortium of GJB2 35delG. Eur J Hum Genet 2000;8:19-23.

27. Lucotte G, Mercier G. Meta-analysis of GJB2 mutation 35delG frequencies in Europe. Genet Test 2001;5:149-152.

28. Morell RJ, Kim HJ, Hood LJ, Goforth L, Friderici K, Fisher R, Van Camp G, Berlin CI, Oddoux C, Ostrer H, Keats B, Friedman TB. Mutations in the connexin 26 gene (GJB2) among Ashkenazi Jews with nonsyndromic recessive deafness. N Engl J Med 1998;339:1500-1505.

29. Van Naarden K, Decoufle P, Caldwell K. Prevalence and characteristics of children with serious hearing impairment in metropolitan Atlanta, 1991-1993. Pediatrics 1999; 103:570-575.

30. Kenna MA, Wu BL, Cotanche DA, Korf BR, Rehm HL. Connexin 26 studies in patients with sensorineural hearing loss. Arch Otolaryngol Head Neck Surg 2001;127: 1037-1042.

31. Green GE, Smith RJ, Bent JP, Cohn ES. Genetic testing to identify deaf newborns. JAMA 2000;284:1245

32. Salvador MQ, Fox MA, Schimmenti LA, Telatar M, Yazdai S, Grody WW. Homozygosity for the connexin $26167 \mathrm{delT}$ mutation in an Ashkenazi Jewish family. Am J Hum Genet 2000;67(Suppl 2):202. 
33. Lerer I, Sagi M, Malamud E, Levi H, Raas-Rothschild A, Abeliovich D. Contribution of connexin 26 mutations to nonsyndromic deafness in Ashkenazi patients and the variable phenotypic effect of the mutation 167delT. Am J Med Genet 2000;95:53-56.

34. Abe S, Usami S, Shinkawa H, Kelley PM, Kimberling WJ. Prevalent connexin 26 gene (GJB2) mutations in Japanese. J Med Genet 2000;37:41-43.

35. Denoyelle F, Weil D, Maw MA, Wilcox SA, Lench NJ, Allen-Powell DR, Osborn AH, Dahl HH, Middleton A, Houseman MJ, Dode C, Marlin S, Boulila-ElGaied A, Grati M, Ayadi H, BenArab S, Bitoun P, Lina-Granade G, Godet J, Mustapha M, Loiselet J, El-Zir E, Aubois A, Joannard A, Petit C, et al. Prelingual deafness: high prevalence of a 30delG mutation in the connexin 26 gene. Hum Mol Genet 1997;6:2173-2177.

36. Morle L, Bozon M, Alloisio N, Latour P, Vandenberghe A, Plauchu H, Collet L, Edery P, Godet J, Lina-Granade G. A novel C202F mutation in the connexin26 gene (GJB2) associated with autosomal dominant isolated hearing loss. J Med Genet 2000; 37:368-370.

37. Tekin M, Arnos KS, Xia XJ, Oelrich MK, Liu XZ, Nance WE, Pandya A. W44C mutation in the connexin 26 gene associated with dominant non-syndromic deafness. Clin Genet 2001;59:269-273.

38. Park HJ, Hahn SH, Chun YM, Park K, Kim HN. Connexin 26 mutations associated with nonsyndromic hearing loss. Laryngoscope 2000;110:1535-1538.

39. Marlin S, Garabedian EN, Roger G, Moatti L, Matha N, Lewin P, Petit C, Denoyelle F. Connexin 26 gene mutations in congenitally deaf children: pitfalls for genetic counseling. Arch Otolaryngol Head Neck Surg 2001;127:927-933.

40. Wilcox SA, Saunders K, Osborn AH, Arnold A, Wunderlich J, Kelly T, Collins V, Wilcox LJ, McKinlay Gardner RJ, Kamarinos M, Cone-Wesson B, Williamson R, Dahl HH. High frequency hearing loss correlated with mutations in the GJB2 gene. Hum Genet 2000;106:399-405.

41. Dahl HH, Saunders K, Kelly TM, Osborn AH, Wilcox S, Cone-Wesson B, Wunderlich JL, Du Sart D, Kamarinos M, Gardner RJ, Dennehy S, Williamson R, Vallance N, Mutton P. Prevalence and nature of connexin 26 mutations in children with nonsyndromic deafness. Med J Aust 2001;175:191-194.

42. Milunsky JM, Maher TA, Yosunkaya E, Vohr BR. Connexin-26 gene analysis in hearing-impaired newborns. Genet Test 2000;4:345-349.

43. Murgia A, Orzan E, Polli R, Martella M, Vinanzi C, Leonardi E, Arslan E, Zacchello F. Cx26 deafness: mutation analysis and clinical variability. J Med Genet 1999;36: $829-832$.

44. Estivill X, Fortina P, Surrey S, Rabionet R, Melchionda S, D’Agruma L, Mansfield E, Rappaport E, Govea N, Mila M, Zelante L, Gasparini P. Connexin-26 mutations in sporadic and inherited sensorineural deafness. Lancet 1998;351:394-398.

45. Wilcox SA, Osborn AH, Allen-Powell DR, Maw MA, Dahl HH, Gardner RJ. Connexin26 deafness in several interconnected families. J Med Genet 1999;36:383-385.

46. Griffith AJ, Chowdhry AA, Kurima K, Hood LJ, Keats B, Berlin CI, Morell RJ, Friedman TB. Autosomal recessive nonsyndromic neurosensory deafness at DFNB1 not associated with the compound-heterozygous GJB2 (connexin 26) genotype M34T/167delT. Am J Hum Genet 2000;67:745-749.

47. Kupka S, Mirghomizadeh F, Haug T, Braun S, Leistenschneider P, Schmitz-Salue C, Arold R, Blin N, Zenner HP, Pfister M. Mutational analysis of the connexin26 gene in sporadic cases of moderate to profound deafness. HNO 2000;48:671-674.

48. Kelsell DP, Wilgoss AL, Richard G, Stevens HP, Munro CS, Leigh IM. Connexin mutations associated with palmoplantar keratoderma and profound deafness in a single family. Eur J Hum Genet 2000;8:469-472.

49. Kelley PM, Harris DJ, Comer BC, Askew JW, Fowler T, Smith SD, Kimberling WJ. Novel mutations in the connexin 26 gene (GJB2) that cause autosomal recessive (DFNB1) hearing loss. Am J Hum Genet 1998;62:792-799.

50. Cucci RA, Prasad S, Kelley PM, Green GE, Storm K, Willcox S, Cohn ES, Van Camp G, Smith RJ. The M34T allele variant of connexin 26. Genet Test 2000;4:335-344.

51. Houseman MJ, Ellis LA, Pagnamenta A, Di WL, Rickard S, Osborn AH, Dahl HH, Taylor GR, Bitner-Glindzicz M, Reardon W, Mueller RF, Kelsell DP. Genetic analysis of the connexin-26 M34T variant: identification of genotype M34T/M34T segregating with mild-moderate non-syndromic sensorineural hearing loss. J Med Genet 2001;38:20-25.

52. Lin D, Goldstein JA, Mhatre AN, Lustig LR, Pfister M, Lalwani AK. Assessment of denaturing high-performance liquid chromatography (DHPLC) in screening for mutations in connexin 26 (GJB2). Hum Mutat 2001;18:42-51.

53. Prasad S, Cucci RA, Green GE, Smith RJ. Genetic testing for hereditary hearing loss: Connexin 26 (GJB2) allele variants and two novel deafness-causing mutations (R32C and 645-648delTAGA). Hum Mutat 2000;16:502-508.

54. Maestrini E, Korge BP, Ocana-Sierra J, Calzolari E, Cambiaghi S, Scudder PM, Hovnanian A, Monaco AP, Munro CS. A missense mutation in connexin26, D66H, causes mutilating keratoderma with sensorineural deafness (Vohwinkel's syndrome) in three unrelated families. Hum Mol Genet 1999;8:1237-1243.

55. Heathcote K, Syrris P, Carter ND, Patton MA. A connexin 26 mutation causes a syndrome of sensorineural hearing loss and palmoplantar hyperkeratosis (MIM 148350). J Med Genet 2000;37:50-51.
56. Richard G, White TW, Smith LE, Bailey RA, Compton JG, Paul DL, Bale SJ. Functional defects of $\mathrm{Cx} 26$ resulting from a heterozygous missense mutation in a family with dominant deaf-mutism and palmoplantar keratoderma. Hum Genet 1998;103: 393-399.

57. Engel-Yeger B, Zaaroura S, Zlotogora J, Shalev S, Hujeirat Y, Carrasquillo M, Barges $\mathrm{S}$, Pratt $\mathrm{H}$. The effects of a connexin 26 mutation-35delG - on oto-acoustic emissions and brainstem evoked potentials: homozygotes and carriers. Hear Res 2002; 163:93-100.

58. Del Castillo I, Villamar M, Moreno-Pelayo MA, del Castillo FJ, Alvarez A, Telleria D, Menendez I, Moreno F. A deletion involving the connexin 30 gene in nonsyndromic hearing impairment. N Engl J Med 2002;346:243-249.

59. Abe S, Kelley PM, Kimberling WJ, Usami SI. Connexin 26 gene (GJB2) mutation modulates the severity of hearing loss associated with the $1555 \mathrm{~A} \rightarrow \mathrm{G}$ mitochondrial mutation. Am J Med Genet 2001;103:334-338.

60. Joint Committee on Infant Hearing. Year 2000 position statement: principles and guidelines for early hearing detection and intervention programs. Am J Audiol 2000; 9:9-29.

61. Kazazian HH, Boehm C, Seltzer WK. ACMG recommendations for standards for interpretation of sequence variation. Genet Med 2000;2:302-303.

62. Holtzman NA, Watson MS, editors. Final report of the Task Force on Genetic Testing: Promoting safe and effective genetic testing in the Unites States. Washington DC: National Human Genome Research Institute, 1997.

63. Secretary's Advisory Committee on Genetic Testing. Enhancing the oversight of genetic tests: recommendations of the SACGT. Bethesda MD: National Institutes of Health, 2000.

64. Cohn ES, Kelley PM, Fowler TW, Gorga MP, Lefkowitz DM, Kuehn HJ, Schaefer GB, Gobar LS, Hahn FJ, Harris DJ, Kimberling WJ. Clinical studies of families with hearing loss attributable to mutations in the connexin 26 gene. Pediatrics 1999;103: $546-550$.

65. Cohn ES, Kelley PM. Clinical phenotype and mutations in connexin 26 (DFNB1/ GJB2), the most common cause of childhood hearing loss. Am J Med Genet 1999;89: $130-136$.

66. American Society of Human Genetics and the American College of Medical Genet ics. Points to consider: Ethical, legal, and psychological implications of genetic testing in children and adolescents. Am J Hum Genet 1995;57:1233-1241.

67. Nance WE, Cunningham GC, Davis JG, Morton CC, Elsas LJ, Finitzo T, Falk RE, Ing PS, Pandya A, McCabe ERB, Smith RJH. Statement of the American College of Medical Genetics on universal newborn hearing screening. Am J Med Genet 2000;2: $149-150$.

68. Middleton A, Hewison J, Mueller RF. Attitudes of deaf adults toward genetic testing for hereditary deafness. Am J Hum Genet 1998;63:1175-1180.

69. Brunger JW, Murray GS, O'Riordan M, Matthews AL, Smith RJ, Robin NH. Parental attitudes toward genetic testing for pediatric deafness. Am J Hum Genet 2000;67: 1621-1625.

70. Rabionet R, Zelante L, Lopez-Bigas N, D’Agruma L, Melchionda S, Restagno G, Arbones ML, Gasparini P, Estivill X. Molecular basis of childhood deafness resulting from mutations in the GJB2 (connexin 26) gene. Hum Genet 2000;106:40-44.

71. Zelante L, Gasparini P, Estivill X, Melchionda S, D’Agruma L, Govea N, Mila M, Monica MD, Lutfi J, Shohat M, Mansfield E, Delgrosso K, Rappaport E, Surrey S, Fortina P. Connexin26 mutations associated with the most common form of nonsyndromic neurosensory autosomal recessive deafness (DFNB1) in Mediterraneans. Hum Mol Genet 1997;6:1605-1609.

72. Bale SJ, White TW, Munro C, Taylor AEM, Richard G. Functional defects of Cx26 due to mutations in two families with dominant palmoplantar keratoderma and deafness. J Invest Dermatol 1999;112:A550.

73. Carrasquillo MM, Zlotogora J, Barges S, Chakravarti A. Two different connexin 26 mutations in an inbred kindred segregating non-syndromic recessive deafness: implications for genetic studies in isolated populations. Hum Mol Genet 1997;6:21632172 .

74. Scott DA, Kraft ML, Carmi R, Ramesh A, Elbedour K, Yairi Y, Srisailapathy CR, Rosengren SS, Markham AF, Mueller RF, Lench NJ, Van Camp G, Smith RJ, Sheffield VC. Identification of mutations in the connexin 26 gene that cause autosomal recessive nonsyndromic hearing loss. Hum Mutat 1998;11:387-394.

75. Erlich PM, Houjeirat Y, Shalev S, Berry COA, Bonne-Tamir B. Hum Genet 1999;104: 529.

76. Brobby GW, Muller-Myhsok B, Horstmann RD. Connexin 26 R143W mutation associated with recessive nonsyndromic sensorineural deafness in Africa. $N$ Engl J Med 1998;338:548-550.

77. Antoniadi T, Gronskov K, Sand A, Pampanos A, Brondum-Nielsen K, Petersen MB. Mutation analysis of the GJB2 (connexin 26) gene by DGGE in Greek patients with sensorineural deafness. Hum Mutat 2000;16:7-12.

78. Storm K, Willocx S, Flothmann K, Van Camp G. Determination of the carrier frequency of the common GJB2 (connexin-26) 35delG mutation in the Belgian 
population using an easy and reliable screening method. Hum Mutat 1999;14:263266.

79. Sartorato EL, Gottardi E, de Oliveira CA, Magna LA, Annichino-Bizzacchi JM, Seixas CA, Maciel-Guerra AT. Determination of the frequency of the 35delG allele in Brazilian neonates. Clin Genet 2000;58:339-340.

80. Lucotte G, Bathelier C, Champenois T. PCR test for diagnosis of the common GJB2 (connexin 26) 35delG mutation on dried blood spots and determination of the carrier frequency in France. Mol Cell Probes 2001;15:57-59.

81. Antoniadi T, Rabionet R, Kroupis C, Aperis GA, Economides J, Petmezakis J, Economou-Petersen E, Estivill X, Petersen MB. High prevalence in the Greek population of the $35 \mathrm{delG}$ mutation in the connexin 26 gene causing prelingual deafness. Clin Genet 1999;55:381-382.

82. Sobe T, Erlich P, Berry A, Korostichevsky M, Vreugde S, Avraham KB, Bonne-Tamir B, Shohat M. High frequency of the deafness-associated 167delT mutation in the connexin 26 (GJB2) gene in Israeli Ashkenazim. Am J Med Genet 1999;86:499-500.

83. Simsek M, Al-Wardy N, Al-Khabory M. A seminested PCR test for simultaneous detection of two common mutations (35delG and 167delT) in the connexin-26 gene. Mol Diagn 2001;6:63-67.

84. Wiszniewski W, Sobieszczanska-Radoszewska L, Nowakowska-Szyrwinska E, Obersztyn E, Bal J. High frequency of GJB2 gene mutations in Polish patients with prelingual nonsyndromic deafness. Genet Test 2001;5:147-148.

85. Anichkina A, Kulenich T, Zinchenko S, Shagina I, Polyakov A, Ginter E, Evgrafov O, Viktorova T, Khusnitdonova E. On the origin and frequency of the $35 \mathrm{delG}$ allele in GJB2-linked deafness in Europe. Eur J Hum Genet 2001;9:151.

86. Masmoudi S, Elgaied-Boulila A, Kassab I, Arab SB, Blanchard S, Bouzouita JE, Drira M, Kassab A, Hachicha S, Petit C, Ayadi H. Determination of the frequency of connexin26 mutations in inherited sensorineural deafness and carrier rates in the Tunisian population using DGGE. J Med Genet 2000;37:E39.

87. Tekin M, Akar N, Cin S, Blanton SH, Xia XJ, Liu XZ, Nance WE, Pandya A. Connexin 26 (GJB2) mutations in the Turkish population: implications for the origin and high frequency of the 35delG mutation in Caucasians. Hum Genet 2001;108: 385-389.

88. Dong J, Katz DR, Eng CM, Kornreich R, Desnick RJ. Nonradioactive detection of the common Connexin 26 167delT and 35delG mutations and frequencies among Ashkenazi Jews. Mol Genet Metab 2001;73:160-163.

89. Lench N, Houseman M, Newton V, Van Camp G, Mueller R. Connexin-26 mutations in sporadic non-syndromal sensorineural deafness. Lancet 1998;351:415.

90. Casademont I, Bizet C, Chevrier D, Guesdon J. Rapid detection of Campylobacter fetus by polymerase chain reaction combined with non-radioactive hybridization using an oligonucleotide covalently bound to microwells. Mol Cell Probes 2000;14: 233-240.

91. Tessa A, Patrono C, Santorelli FM, Giannotti A, Digilio MC, Pacifico C, Presuttari F, Tieri L. Rapid detection of the 35delG mutation in the GJB2 gene in childhood deafness. J Med Screen 2000;7:167.

92. Angeli S, Utrera R, Dib S, Chiossone E, Naranjo C, Henriquez O, Porta M. GJB2 gene mutations in childhood deafness. Acta Otolaryngol 2000;120:133-136.

\section{APPENDIX}

\section{Statements}

- National Institute on Deafness and Other Communication Disorders (NIDCD) Working Group Considerations for Developing and Implementing Genetic Diagnostic Tests for Hereditary Hearing Impairment and Other Communication Disorders (December 1998): www.nidcd.nih.gov/textonly/funding/hb/ genetic.htm

- Statement of the American College of Medical Genetics on Universal Newborn Hearing Screening (January 2000): www.faseb. org/genetics/acmg/pol-35.htm

\section{Links}

Hearing and hearing loss resources

- Connexin 26 Homepage: www.iro.es/deafness/

- GeneClinics: Deafness Overview: www.geneclinics.org/ profiles/deafness-overview/details.html

- Hereditary Hearing Loss Homepage: dnalab-www.uia. ac.be/dnalab/hhh/index.html

- National Institute on Deafness and Other Communication Disorders: www.nidcd.nih.gov/health/hb.htm

- Promenade 'round the Cochlea: www.iurc.montp.inserm. $\mathrm{fr} /$ cric/audition/english/index.htm

- The Genetics of Infant Hearing Loss: www.cdc.gov/ncbddd/ ehdi/genetics.htm

\section{General resources}

- Online Mendelian Inheritance in Man: www.ncbi.nlm.nih .gov/entrez/query.fcgi? db=OMIM

- GenBank: www.ncbi.nlm.nih.gov/Genbank/Genbank Overview.html

- Human Gene Mutation Database: archive.uwcm.ac.uk/ uwcm/mg/hgmd0.html 